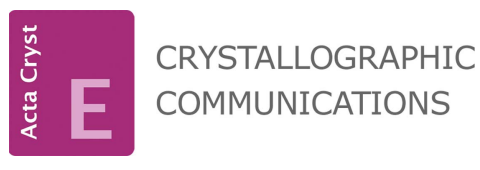

ISSN 2056-9890

Received 16 October 2018

Accepted 15 November 2018

Edited by B. Therrien, University of Neuchâtel, Switzerland

Keywords: crystal structure; Hirshfeld surface analysis; fingerprint plots; Schiff bases; $(E)-N^{\prime}$-[4(piperidin-1-yl)benzylidene]arylsulfonohydrazides.

CCDC references: $1879247 ; 1879246$; 1879245

Supporting information: this article has supporting information at journals.iucr.org/e

\section{Crystal structure and Hirshfeld surface analysis of (E)- $N^{\prime}$-[4-(piperidin-1-yl)benzylidene]arylsulfono- hydrazides}

\author{
Nikhila Pai, ${ }^{a}$ Sabine Foro ${ }^{\mathrm{b}}$ and B. Thimme Gowda ${ }^{\mathrm{a}, \mathrm{c} *}$ \\ ${ }^{\mathbf{a}}$ Department of Chemistry, Mangalore University, Mangalagangotri-574 199, Mangalore, India, ${ }^{\mathbf{b}}$ Institute of Materials \\ Science, Darmstadt University of Technology, Alarich-Weiss-Str. 2, D-64287, Darmstadt, Germany, and ${ }^{\mathbf{c}}$ Karnataka State \\ Rural Development and Panchayat Raj University, Gadag-582 101, India. *Correspondence e-mail: \\ gowdabt@yahoo.com
}

The crystal structures and Hirshfeld surface analyses of three Schiff bases, namely (E)- $N^{\prime}$-[4-(piperidin-1-yl)benzylidene]benzenesulfonohydrazide, $\mathrm{C}_{18} \mathrm{H}_{21} \mathrm{~N}_{3} \mathrm{O}_{2} \mathrm{~S}$, (I), (E)-4-methyl- $N^{\prime}$-[4-(piperidin-1-yl)benzylidene]benzenesulfonohydrazide, $\mathrm{C}_{19} \mathrm{H}_{23} \mathrm{~N}_{3} \mathrm{O}_{2} \mathrm{~S}$, (II), and (E)-4-chloro- $N^{\prime}$-[4-(piperidin-1yl)benzylidene]benzenesulfonohydrazide, $\mathrm{C}_{18} \mathrm{H}_{20} \mathrm{ClN}_{3} \mathrm{O}_{2} \mathrm{~S}$, (III), derived from arylsulfonohydrazides and 4-(piperidin-4-yl)benzaldehyde have been analysed to investigate the effect of substituents on the structural parameters. All three structures crystallize in monoclinic crystal systems, in the space groups $P 2_{1} / c$ for (I) and (II), and $C 2 / c$ for (III). Compound (III) contains two independent molecules in the asymmetric unit and sixteen molecules per unit cell, while (I) and (II) both have one and four molecules, respectively, in their asymmetric units and unit cells. In all cases, the central part of the molecule is twisted at the $\mathrm{S}$ atom. In the crystals, the molecules are linked via $\mathrm{N}-\mathrm{H} \cdots \mathrm{O}$ hydrogen bonds, forming chains. Two-dimensional fingerprint plots of various interatomic contacts show that the major contributions are from $\mathrm{H} \cdots \mathrm{H}$ interactions.

\section{Chemical context}

Piperidine is very common in many natural and synthetic Ncontaining medicaments and is present in the basic skeleton of many pharmacologically active compounds (Sampath, 2017). Compounds with a piperidine functional group are intermediates in the synthesis of various alkaloids (Wang \& Wuorola, 1992; Grishina et al., 1995). They are reported to be cholesterol-lowering (Comins et al., 2001) and to display antiviral (Kang et al., 2015), anti-inflammatory, antioxidant (Tharini \& Sangeetha, 2015), anti-epileptic (Kiasalari et al., 2014), antimicrobial, antitumor and antifungal (Sahu et al., 1979; Shah et al., 1992) activities. Furthermore, Schiff bases find applications in the pharmacological field and are important in designing medicines (Parekh et al., 2005). Thus the crystal structures of Schiff bases and piperidine derivatives have always been interesting, especially with regard to the stereochemistry across $\mathrm{C}=\mathrm{N}$ and the conformation of the sixmembered heterocyclic ring. We were interested in exploring the effect of the substituents on the structural parameters of compounds containing these moieties. Thus we report herein the synthesis, characterization and crystal structures of $(E)-N^{\prime}$ [4-(piperidin-1-yl)benzylidene] benzenesulfonohydrazide, $\mathrm{C}_{18} \mathrm{H}_{21} \mathrm{~N}_{3} \mathrm{O}_{2} \mathrm{~S}$, (I), and its 4-methyl- and 4-chloro-derivatives, 
namely, (E)-4-methyl- $N^{\prime}$-[4-(piperidin-1-yl)benzylidene]benzenesulfonohydrazide, $\mathrm{C}_{19} \mathrm{H}_{23} \mathrm{~N}_{3} \mathrm{O}_{2} \mathrm{~S}$, (II), and (E)-4-chloro- $N^{\prime}$ [4-(piperidin-1-yl)benzylidene]benzenesulfonohydrazide, $\mathrm{C}_{18} \mathrm{H}_{20} \mathrm{ClN}_{3} \mathrm{O}_{2} \mathrm{~S}$, (III).<smiles>[R]c1ccc(S(=O)(=O)N/N=C/c2ccc(N3CCCCC3)cc2)cc1</smiles>

$$
\text { (I) } R=\mathrm{H} \text {, (II) } R=\mathrm{CH}_{3} \text {, (III) } R=\mathrm{Cl}
$$

\section{Structural commentary}

All three of the title compounds (Figs. 1-3) crystallize in the monoclinic crystal system but in space group $P 2_{1} / c$ for (I) and (II), and space group $C 2 / c$ for (III). The asymmetric units of compounds (I) and (II) each contain one molecule whereas there are two independent molecules in the asymmetric unit of (III). All the three compounds display an $E$-configuration about the $\mathrm{C}=\mathrm{N}$ bond (Purandara et al., 2017; Gu et al., 2012), and a chair conformation of the piperidine ring.

In compounds (I) and (II) (Figs. 1 and 2), the sulfonamide bonds are found to be synclinal and the torsion angles of the sulfonamide moieties are $-66.0(2)$ and $63.5(2)^{\circ}$, respectively (Moss, 1996). The dihedral angles between the phenyl ring (C1-C6/S1) and the mean plane of the N1/N2/C7-C9 hydrazone fragment are $85.3(1)$ and $80.5(1)^{\circ}$ in (I) and (II), respectively, indicating that the hydrazone portion of the molecules $(\mathrm{C}=\mathrm{N}-\mathrm{N}-\mathrm{S}-\mathrm{C}$ group $)$ is not coplanar with the sulfonyl phenyl ring. The $\mathrm{C} 7=\mathrm{N} 2$ bond lengths of 1.271 (3) $\AA$ in (I) and 1.269 (3) $\AA$ in (II) are in agreement with doublebond character. In both compounds, the piperidine group is not sterically hindered. Thus the six-membered heterocyclic ring adopts the most stable chair conformation. The total puckering amplitude is 0.531 (3) $\AA$ in (I) and 0.465 (4) $\AA$ in (II), the puckering parameters are 173.7 (3), and $8.0(5)^{\circ}$ in (I) and (II), respectively, and the phase angles are 13.0 (3) in (I) and $184.0(4)^{\circ}$ in (II), respectively (Cremer \& Pople, 1975; Nardelli, 1983). The C15-C14-N3-C11 torsion angles of

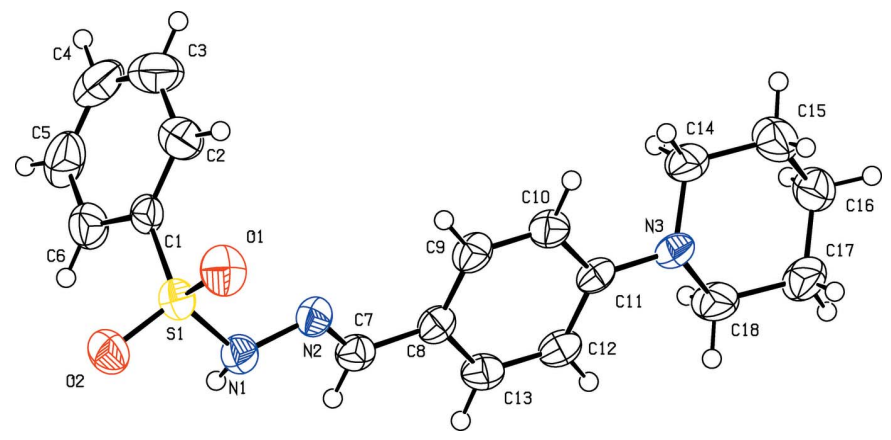

Figure 1

Molecular structure of (I), showing the atom labelling and displacement ellipsoids drawn at the $50 \%$ probability level.

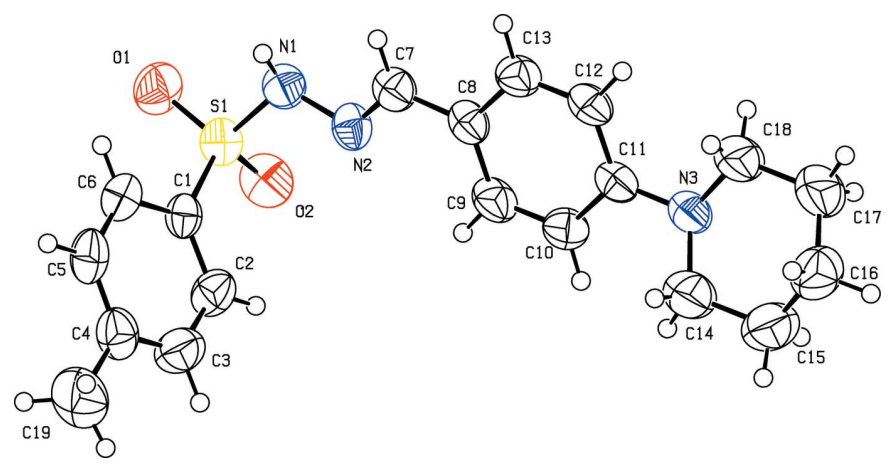

Figure 2

Molecular structure of (II), showing the atom labelling and displacement ellipsoids drawn at the $50 \%$ probability level.

$-172.2(2)^{\circ}$ and $175.2(3)^{\circ}$ in (I) and (II), respectively, signify that the phenyl ring at the $\mathrm{N}$ atom of the piperidine ring is in an equatorial position (Nallini et al., 2003).

The asymmetric unit of (III) contains two independent molecules and the unit cell contains 16 molecules. The torsion angles for the sulfonamide moieties in the two molecules $\left[\mathrm{C} 1-\mathrm{S} 1-\mathrm{N} 1-\mathrm{N} 2=59.7(4)^{\circ}\right.$ and $\mathrm{C} 19-\mathrm{S} 2-\mathrm{N} 4-\mathrm{N} 5=$ $\left.67.9(4)^{\circ}\right]$ signify a synclinal conformation (Moss, 1996). The hydrazone moiety $(\mathrm{C}=\mathrm{N}-\mathrm{N}-\mathrm{S}-\mathrm{C}$ group $)$ and arylsulfonyl ring are not coplanar, with dihedral angles between the two planes of $87.3(1)$ and $79.4(1)^{\circ}$, respectively, in the first and second molecules. The $\mathrm{C} 7=\mathrm{N} 2$ and $\mathrm{C} 25=\mathrm{N} 5$ bond lengths of 1.272 (5) and $1.269(5) \AA$, respectively, are consistent with double-bond character. As in compounds (I) and (II), the piperidine group in (III) adopts a chair conformation, with the total puckering amplitude of $Q_{T}=0.283$ (7) and 0.475 (1) $\AA$ in the first and second molecules, respectively, $\theta=2.7$ (14), $175.5(8)^{\circ}$ and phase angles $\varphi=220(22)^{\circ}$ and $353(10)^{\circ}$ in the two molecules, respectively. The phenyl ring at the piperidine $\mathrm{N}$ atom is equatorial, as is evident from $\mathrm{C} 15-\mathrm{C} 14-\mathrm{N} 3-\mathrm{C} 11$ and $\mathrm{C} 33-\mathrm{C} 32-\mathrm{N} 6-\mathrm{C} 29$ torsion angles of $174.4(7)$ and $-168.9(5)^{\circ}$, respectively.

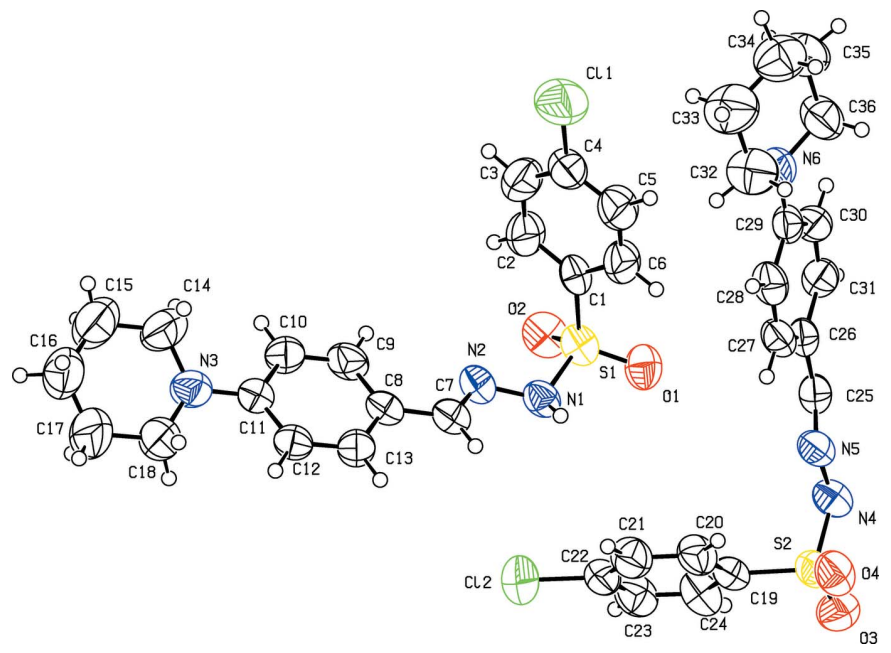

Figure 3

Molecular structure of (III), showing the atom labelling and displacement ellipsoids drawn at the $50 \%$ probability level. 

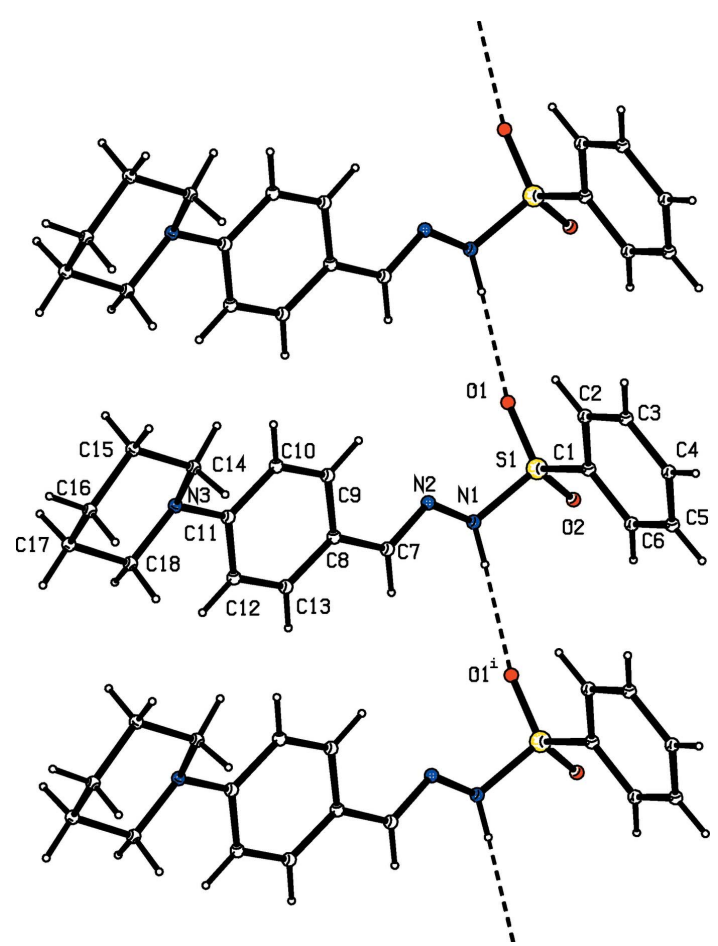

Figure 4

Hydrogen-bonding pattern in (I) with hydrogen bonds shown as dashed lines. Symmetry code as in Table 1.

\section{Supramolecular features}

In all the three crystal structures, the amino $\mathrm{H}$ atom of the sulfonohydrazide segment acts as a donor and the sulfonyl $\mathrm{O}$ atom acts as an acceptor in $\mathrm{N}-\mathrm{H}$... O hydrogen-bonding interactions that generate $C 4$ chains propagating parallel to the $b$ axis (Tables 1-3, Figs. 4-9). Substitution at the para position by a methyl or chloro group to produce compounds

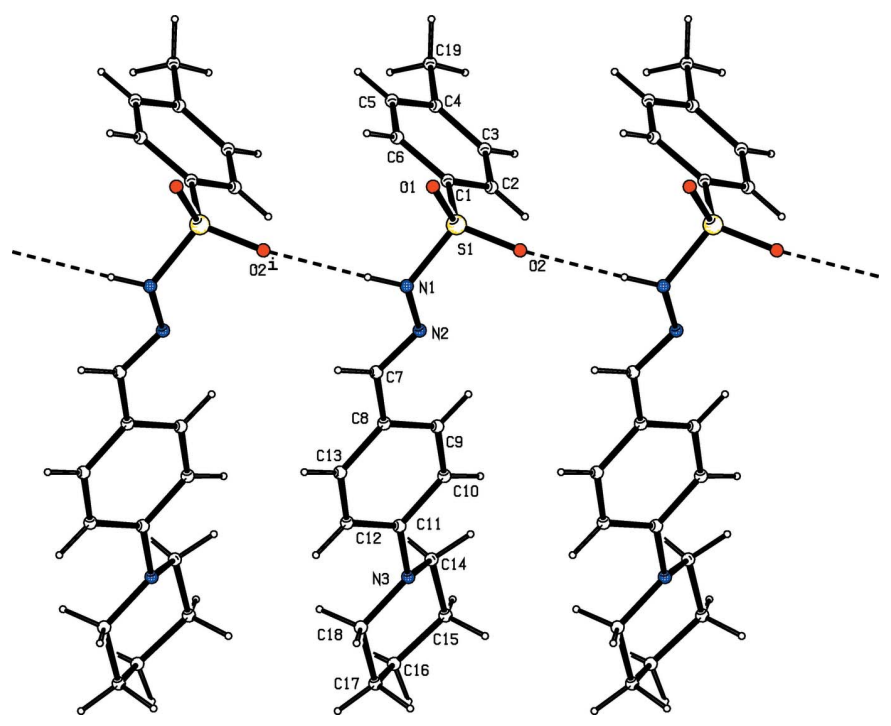

Figure 5

Hydrogen-bonding pattern in (II) with hydrogen bonds shown as dashed lines. Symmetry code as in Table 2.

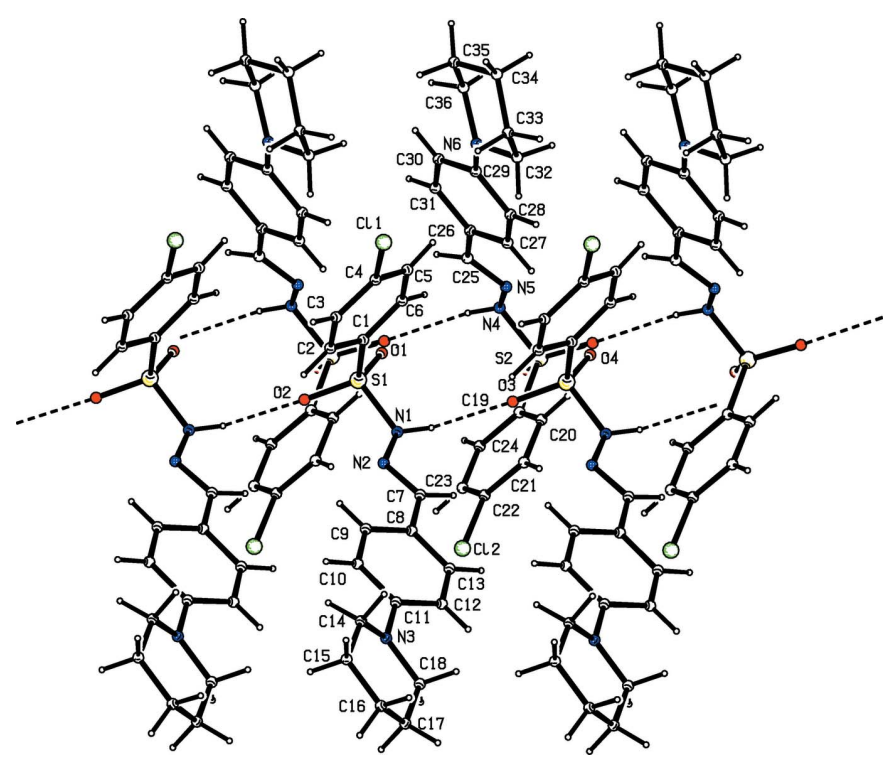

Figure 6

Hydrogen-bonding pattern in (III) with hydrogen bonds shown as dashed lines.

(II) and (III) has no remarkable effect on the hydrogenbonding pattern.

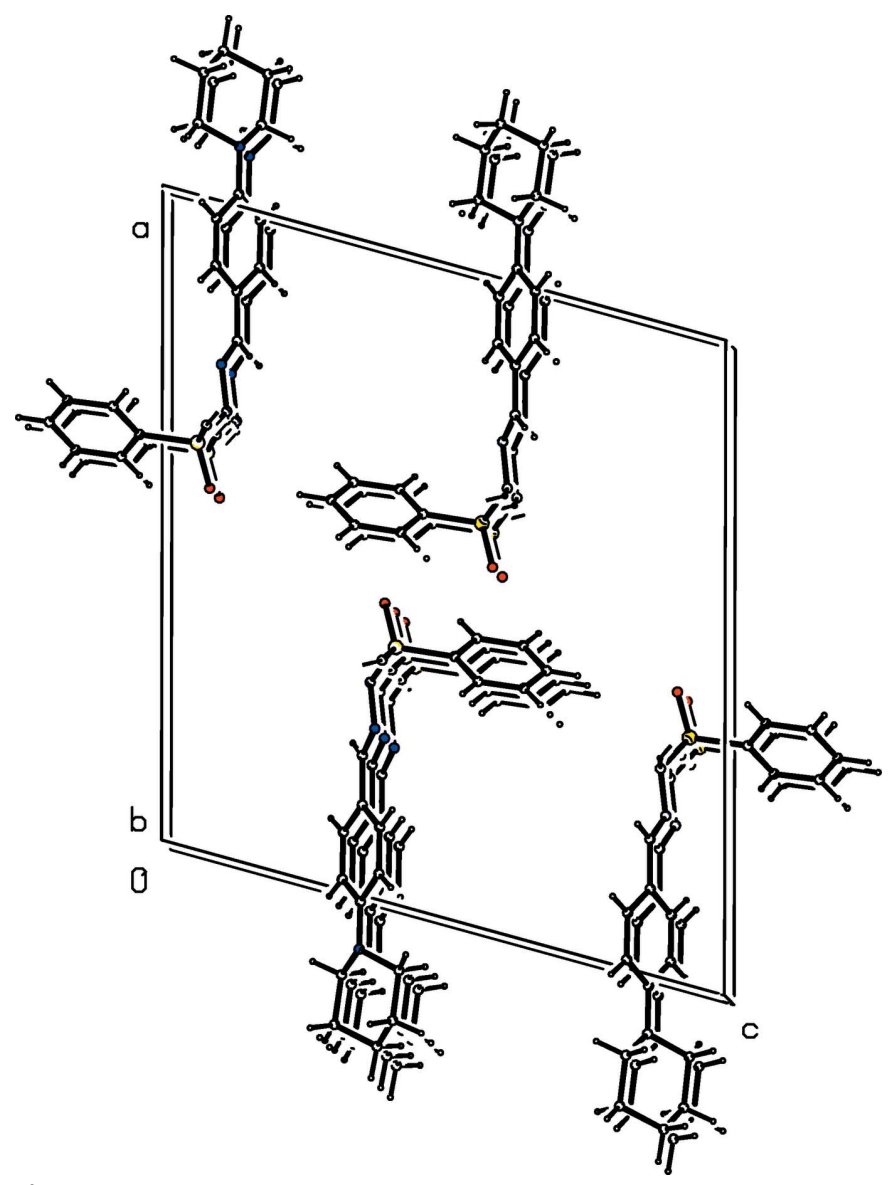

Figure 7

Molecular packing of (I). 
Table 1

Hydrogen-bond geometry $\left(\AA{ }^{\circ}{ }^{\circ}\right)$ for (I).

\begin{tabular}{lllll}
\hline$D-\mathrm{H} \cdots A$ & $D-\mathrm{H}$ & $\mathrm{H} \cdots A$ & $D \cdots A$ & $D-\mathrm{H} \cdots A$ \\
\hline $\mathrm{N} 1-\mathrm{H} 1 N \cdots \mathrm{O} 1^{\mathrm{i}}$ & $0.84(2)$ & $2.32(2)$ & $3.133(3)$ & $165(2)$ \\
\hline
\end{tabular}

Symmetry code: (i) $x, y+1, z$.

Table 2

Hydrogen-bond geometry $\left(\AA,^{\circ}\right)$ for $(\mathrm{II})$.

\begin{tabular}{lllll}
\hline$D-\mathrm{H} \cdots A$ & $D-\mathrm{H}$ & $\mathrm{H} \cdots A$ & $D \cdots A$ & $D-\mathrm{H} \cdots A$ \\
\hline $\mathrm{N} 1-\mathrm{H} 1 N \cdots \mathrm{O} 2^{\mathrm{i}}$ & $0.79(3)$ & $2.29(3)$ & $3.068(3)$ & $170(3)$ \\
\hline
\end{tabular}

Symmetry code: (i) $x, y+1, z$.

Table 3

Hydrogen-bond geometry $\left(\AA,^{\circ}\right)$ for (III).

\begin{tabular}{lllll}
\hline$D-\mathrm{H} \cdots A$ & $D-\mathrm{H}$ & $\mathrm{H} \cdots A$ & $D \cdots A$ & $D-\mathrm{H} \cdots A$ \\
\hline $\mathrm{N} 1-\mathrm{H} 1 N \cdots \mathrm{O} 2^{\mathrm{i}}$ & $0.83(4)$ & $2.26(5)$ & $3.025(5)$ & $153(5)$ \\
$\mathrm{N} 4-\mathrm{H} 4 N \cdots \mathrm{O} 4{ }^{\mathrm{ii}}$ & $0.84(5)$ & $2.29(5)$ & $3.115(6)$ & $169(5)$ \\
\hline
\end{tabular}

Symmetry codes: (i) $x, y+1, z$; (ii) $x, y-1, z$.

\section{Database survey}

Although there are several reports on the crystal structures of piperidine or sulfonylhydrazides derivatives, reports on the crystal structures of 4-(piperidin-1-yl)benzaldehyde functionalized with sulfonylhydrazides are very few. Comparison of

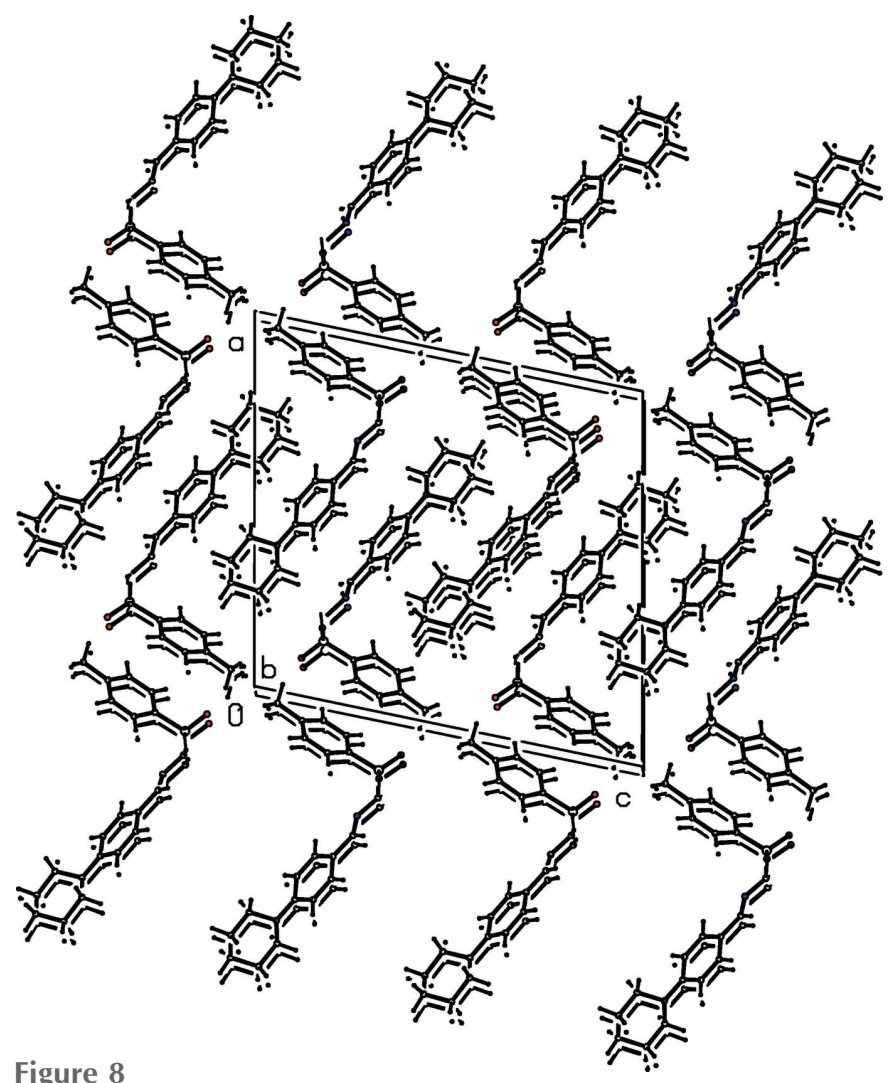

Molecular packing of (II).

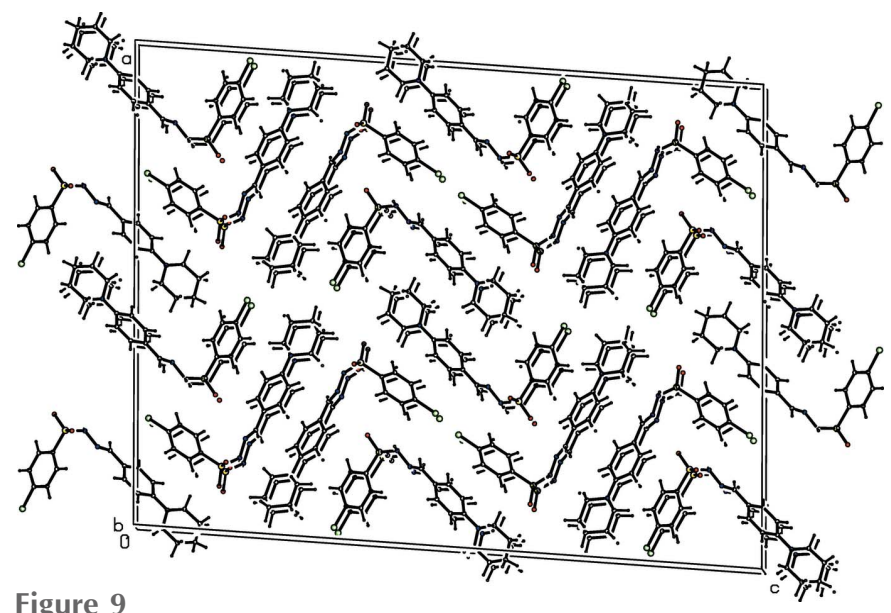

Figure 9

Molecular packing of (III).

the present data with those of thiophene/phenyl-piperidine hybrid chalcones (Parvez et al., 2014) reveals that the compounds also adopt $E$ configuration around the $\mathrm{C}=\mathrm{N}$ bond and the piperidine rings exhibit a chair conformation. A chair conformation of the piperidine ring is also found in 5-nitro-2(piperidin-1-yl)benzaldehyde (N'Gouan et al., 2009) and (5-nitro-2-piperidino)benzylidene $\quad p$-toluenesulfonylhydrazone (Yapo et al., 2008).

\section{Hirshfeld surface analysis}

Hirshfeld surfaces (HS) and 2D fingerprint plots were generated using CrystalExplorer17 (Turner et al., 2017; McKinnon et al., 2007; Spackman \& Jayatilaka, 2009). The terms such as $d_{\text {norm }}, d_{\mathrm{i}}$ and $d_{\mathrm{e}}$ are defined in the usual way (Shit et al., 2016). The function $d_{\text {norm }}$ is a ratio enclosing the distances of any surface point to the nearest interior $\left(d_{\mathrm{i}}\right)$ and exterior $\left(d_{\mathrm{e}}\right)$ atom and the van der Waals radii of the atoms (Hirshfeld, 1977; Soman et al., 2014). The function $d_{\text {norm }}$ will be equal to zero when intermolecular distances are close to van der Waals contacts. They are indicated by a white colour on the HS, while contacts longer than the sum of van der Waals radii with positive $d_{\text {norm }}$ values are coloured in blue. The surface images and plots for $d_{\text {norm }}$ (Fig. 10) were generated using a high standard surface resolution over a colour scale of -0.3495 to $1.3559,-0.4124$ to 1.6768 and -0.3876 to 1.5649 a.u. for (I), (II) and (III), respectively.

Hirshfeld fingerprint plots for various interactions show differences in the percentage contributions to the Hirshfeld surfaces. $\mathrm{H} \cdots \mathrm{H}$ contacts make the maximum contribution to the Hirshfeld surfaces in all three compounds. The contributions of significant contacts in the three compounds are in the following order: $\mathrm{H} \cdots \mathrm{H}, \mathrm{C} \cdots \mathrm{H} / \mathrm{H} \cdots \mathrm{C}$ and $\mathrm{O} \cdots \mathrm{H} / \mathrm{H} \cdots \mathrm{O}$. In compound (I), these interactions cover a region of $52.0 \%\left(d_{\mathrm{i}}=\right.$ $\left.d_{\mathrm{e}}=1.5 \AA\right), 22.5 \%\left(d_{\mathrm{i}}+d_{\mathrm{e}}=3.2 \AA\right)$, and $15.3 \%\left(d_{\mathrm{i}}+d_{\mathrm{e}}=\right.$ $2.4 \AA$ A) (Fig. 11), respectively. The other interatomic contacts and percentages of contributions to the Hirshfeld surface are $\mathrm{N} \cdots \mathrm{H} / \mathrm{H} \cdots \mathrm{N}(6.7 \%), \mathrm{C} \cdots \mathrm{O} / \mathrm{O} \cdots \mathrm{C}(3.1 \%)$. In compound (II), the contributions of the various contacts are: $\mathrm{H} \cdots \mathrm{H}$ $52.3 \%\left(d_{\mathrm{i}}=d_{\mathrm{e}}=1.5 \AA\right), \mathrm{C} \cdots \mathrm{H} / \mathrm{H} \cdots \mathrm{C} 23.6 \%\left(d_{\mathrm{i}}+d_{\mathrm{e}}=3.2 \AA\right)$, 


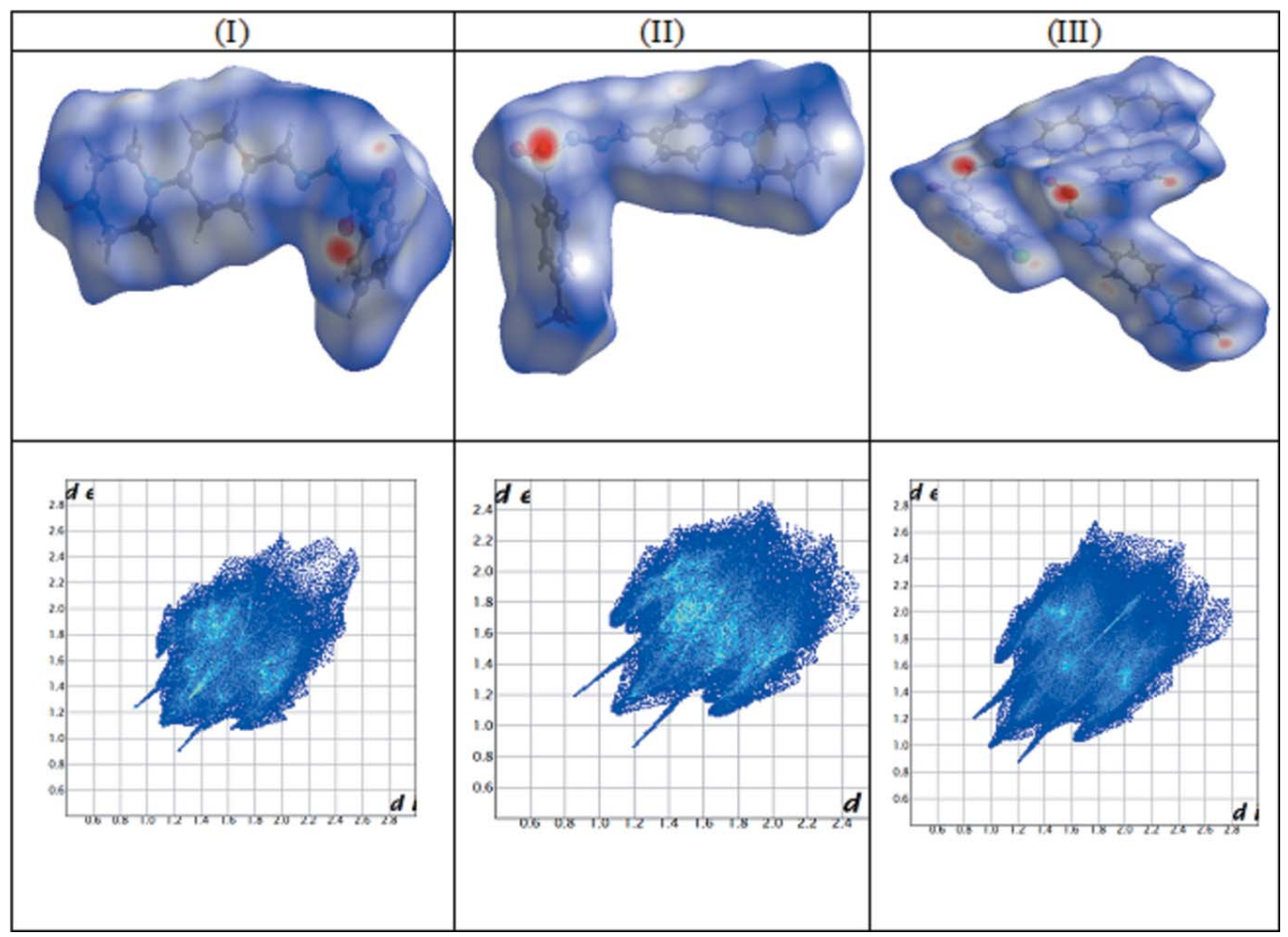

Figure 10

Top: Hirshfeld surface mapped over $d_{\text {norm }}$ for (I), (II) and (III). Bottom: two-dimensional fingerprint plots for (I), (II) and (III).

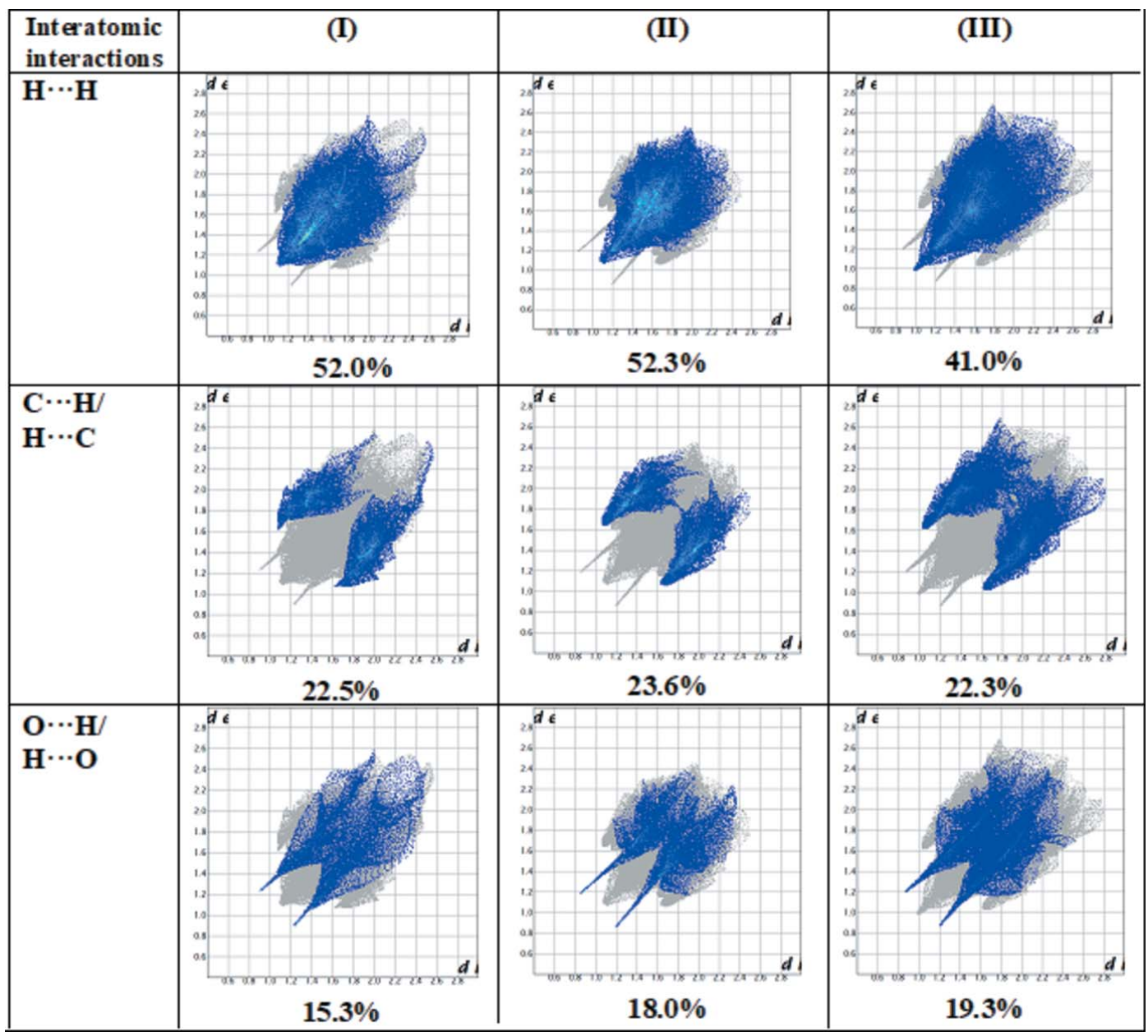

Figure 11

Two-dimensional fingerprint plots for (I), (II) and (III), showing the contributions of the different types of interactions. 
and $\mathrm{O} \cdots \mathrm{H} / \mathrm{H} \cdots \mathrm{O} 18.0 \%\left(d_{\mathrm{i}}+d_{\mathrm{e}}=2.4 \AA\right)$ (Fig. 11). Among the minor contributions observed, $\mathrm{N} \cdots \mathrm{H} / \mathrm{H} \cdots \mathrm{N}$ interaction cover a region of $6.1 \%$. In the case of compound (III), the major contributions are $\mathrm{H} \cdots \mathrm{H} 41.0 \%\left(d_{\mathrm{i}}=d_{\mathrm{e}}=1.0 \AA\right), \mathrm{C} \cdots \mathrm{H} /$ $\mathrm{H} \cdots \mathrm{C} 22.3 \%(3.2 \AA)$ and $\mathrm{O} \cdots \mathrm{H} / \mathrm{H} \cdots \mathrm{O}, 19.3 \%\left(d_{\mathrm{i}}+d_{\mathrm{e}}=\right.$ $2.4 \AA$ ) along with minor contributions from $\mathrm{Cl} \cdots \mathrm{H} / \mathrm{H} \cdots \mathrm{Cl}$ $(9.5 \%)$ and $\mathrm{N} \cdots \mathrm{H} / \mathrm{H} \cdots \mathrm{N}(5.1 \%)$ interactions (Fig. 11).

\section{Synthesis and crystallization}

Synthesis of benzenesulfonohydrazide and 4-methyl and 4-chloro-benzenesulfonohydrazides

To solutions of hydrazine hydrate $(99 \%)(0.03 \mathrm{~mol})$ in THF at $273 \mathrm{~K}$ under stirring, a solution of benzenesulfonyl chloride, 4-methylbenzenesulfonyl chloride or 4-chlorobenzenesulfonyl chloride $(0.02 \mathrm{~mol})$ in THF was added dropwise. Three separate reaction mixtures were kept under stirring at $273 \mathrm{~K}$ for $1 \mathrm{~h}$ and stirring continued for $24 \mathrm{~h}$ at room temperature. The formation of the products was monitored by TLC. After completion of the reactions, the reaction mixtures were poured separately onto ice-cold water. The separated solids, benzenesulfonohydrazide, 4-methylbenzenesulfonohydrazide or 4-chlorobenzenesulfonohydrazide, were filtered off and dried. The products were recrystallized from ethanol solution to get the pure products.

The purity of the compounds was checked by TLC and they were characterized by their IR spectra. They were further characterized by ${ }^{1} \mathrm{H}$ and ${ }^{13} \mathrm{C}$ NMR spectra. The characteristic IR absorptions and ${ }^{1} \mathrm{H}$ and ${ }^{13} \mathrm{C}$ NMR signals are as follows:

Benzenesulfonohydrazide: m.p. 374-376 K; FT-IR (ATR, $\left.v_{\max }, \mathrm{cm}^{-1}\right): 3254.4\left(s, \mathrm{NH}_{2} \mathrm{str}\right), 3198.3(s, \mathrm{~N}-\mathrm{H}$ str), $1325.1(s$, $\mathrm{S}=\mathrm{O}$ asym str) and 1140.8 ( $v s, \mathrm{~S}=\mathrm{O}$ sym str).

${ }^{1} \mathrm{H}$ and ${ }^{13} \mathrm{C}$ NMR spectra: ${ }^{1} \mathrm{H}\left(400 \mathrm{MHz}\right.$, DMSO- $d_{6}, \delta$, ppm): 7.93-7.42 (m, 5H, Ar-H), $5.85(t, 1 \mathrm{H}), 3.43(d, 2 \mathrm{H}) .{ }^{13} \mathrm{C} \mathrm{NMR}$ (100 MHz, DMSO- $\left.d_{6}, \delta, \mathrm{ppm}\right) ; 134.57,130.15,129.12,125.63$.

4-Methylbenzenesulfonohydrazide: m.p. 382-385 K; FT-IR (ATR, $\left.v_{\max }, \mathrm{cm}^{-1}\right): 3245.1\left(s, \mathrm{NH}_{2} \mathrm{str}\right), 3193.8(s, \mathrm{~N}-\mathrm{H}$ str), 1330.5 ( $s, \mathrm{~S}=\mathrm{O}$ asym str) and 1126.5 ( $v s, \mathrm{~S}=\mathrm{O}$ sym str).

${ }^{1} \mathrm{H}$ and ${ }^{13} \mathrm{C}$ NMR spectra: ${ }^{1} \mathrm{H}\left(400 \mathrm{MHz}\right.$, DMSO- $d_{6}, \delta$, ppm); 7.71-7.31 (m, 4H, Ar-H), $5.91(t, 1 \mathrm{H}), 3.48(d, 2 \mathrm{H}), 2.19(s$, $\left.3 \mathrm{H}, \mathrm{CH}_{3}\right) .{ }^{13} \mathrm{C}$ NMR $\left(100 \mathrm{MHz}, \mathrm{DMSO}-d_{6}, \delta, \mathrm{ppm}\right) ; 142.36$, 136.90, 128.13, 126.71, 22.11.

4-Chlorobenzenesulfonohydrazide: m.p. 388-90 K; FT-IR (ATR, $\left.v_{\max }, \mathrm{cm}^{-1}\right): 3259.4\left(s, \mathrm{NH}_{2} \mathrm{str}\right), 3195.1(s, \mathrm{~N}-\mathrm{H}$ str), 1341.7 ( $s, \mathrm{~S}=\mathrm{O}$ asym str) and 1138.5 ( $v s, \mathrm{~S}=\mathrm{O}$ sym str).

${ }^{1} \mathrm{H}$ and ${ }^{13} \mathrm{C}$ NMR spectra: ${ }^{1} \mathrm{H}\left(400 \mathrm{MHz}\right.$, DMSO- $d_{6}, \delta$, ppm); 7.58-7.67 (m, 5H, Ar-H), $5.87(t, 1 \mathrm{H}), 3.41(d, 2 \mathrm{H}) .{ }^{13} \mathrm{C} \mathrm{NMR}$ (100 MHz, DMSO-d 6 , $\delta$, ppm); 137.90, 137.29, 130.30, 128.42.

\section{Synthesis of the title compounds (I), (II) and (III):}

Mixtures of 4-(piperidin-1-yl)benzaldehyde $(0.001 \mathrm{~mol})$ and benzenesulfonohydrazide, 4-methylbenzenesulfonohydrazide or 4-chlorobenzenesulfonohydrazide $(0.001 \mathrm{~mol})$ in ethanol $(10 \mathrm{ml})$ and two drops of glacial acetic acid were stirred at room temperature for $2 \mathrm{~h}$. The formation of the products was monitored by TLC. The reaction mixtures were separately poured on crushed ice and the solids that formed were washed and dried. The products were recrystallized to constant melting points from an acetonitrile:DMF (5:1 v:v) mixture. The purity of the compounds was checked by TLC and they were characterized by their IR spectra. They were further characterized by ${ }^{1} \mathrm{H}$ and ${ }^{13} \mathrm{C}$ NMR spectra. The characteristic IR absorptions and ${ }^{1} \mathrm{H}$ and ${ }^{13} \mathrm{C}$ NMR signals are as follows

Compound (I): m.p. 417-419 K; FT-IR (ATR, $v_{\max }, \mathrm{cm}^{-1}$ ): $3219.2(s, \mathrm{~N}-\mathrm{H}$ str), $1609.3(s, \mathrm{C}=\mathrm{N}$ str), $1363.7(s, \mathrm{~S}=\mathrm{O}$ asym str) and 1165.0 ( $v s, \mathrm{~S}=\mathrm{O}$ sym str).

${ }^{1} \mathrm{H}$ and ${ }^{13} \mathrm{C}$ NMR spectra: ${ }^{1} \mathrm{H}\left(400 \mathrm{MHz}\right.$, DMSO- $d_{6}, \delta$, ppm); $9.41(s, 1 \mathrm{H}, \mathrm{N}-\mathrm{H}), 8.39(s, 1 \mathrm{H},=\mathrm{C}-\mathrm{H}), 7.76-7.59(\mathrm{~m}, 5 \mathrm{H}$, $\mathrm{Ar}-\mathrm{H}), 7.54-6.54(m, 4 \mathrm{H}, \mathrm{Ar}-\mathrm{H}), 3.46-1.82(m, 4 \mathrm{H}), 1.47-$ $1.39(m, 6 \mathrm{H}) .{ }^{13} \mathrm{C}$ NMR $\left(100 \mathrm{MHz}, \mathrm{DMSO}-d_{6}, \delta, \mathrm{ppm}\right) ; 151.34$, $147.31,138.89,133.62,130.94,129.91,128.27,124.97,112.76$, 48.54, 24.82, 23.93.

Compound (II): m.p. 439 - $441 \mathrm{~K}$; FT-IR (ATR, $v_{\max }$, $\left.\mathrm{cm}^{-1}\right)$ : $3214.3(s, \mathrm{~N}-\mathrm{H}, \mathrm{str}), 1606.7(s, \mathrm{C}=\mathrm{N}$ str), $1359.82(s$, $\mathrm{S}=\mathrm{O}$ asym) and $1163.08 \mathrm{~cm}^{-1}(v s, \mathrm{~S}=\mathrm{O}$ sym $)$.

${ }^{1} \mathrm{H}$ and ${ }^{13} \mathrm{C}$ spectra: ${ }^{1} \mathrm{H}\left(400 \mathrm{MHz}\right.$, DMSO- $\left.d_{6}, \delta, \mathrm{ppm}\right) ; 10.98$ $(s, 1 \mathrm{H}, \mathrm{N}-\mathrm{H}), 7.77-7.75(m, 3 \mathrm{H}, \mathrm{Ar}-\mathrm{H},=\mathrm{C}-\mathrm{H}), 7.37-7.34$ $(m, 4 \mathrm{H}, \mathrm{Ar}-\mathrm{H}), 3.29-2.36(m, 4 \mathrm{H}), 2.37\left(s, 3 \mathrm{H}, \mathrm{CH}_{3}\right), 1.56-$ $1.47(m, 6 \mathrm{H}) ;{ }^{13} \mathrm{C}$ NMR (100 MHz, DMSO- $\left.d_{6} \delta, \mathrm{ppm}\right) ; 152.28$, $147.48,142.92,136.34,129.28,127.89,127.17,114.45,48.51$, 24.92, 23.87, 21.0.

Compound (III): m.p. 429-431 K; FT-IR (ATR, v $\max$, $\left.\mathrm{cm}^{-1}\right)$ : $3213.4(s, \mathrm{~N}-\mathrm{H}, \mathrm{str}), 1608.9(s, \mathrm{C}=\mathrm{N}, \mathrm{str}), 1365.6(s$, $\mathrm{S}=\mathrm{O}$ asym str) and 1166.9 ( $v s, \mathrm{~S}=\mathrm{O}$ sym str).

${ }^{1} \mathrm{H}$ and ${ }^{13} \mathrm{C}$ spectra: ${ }^{1} \mathrm{H}$ (400 MHz, DMSO- $d_{6}, \delta$, ppm); 8.18 $(s, 1 \mathrm{H}, \mathrm{N}-\mathrm{H}), 7.91-7.88(m, 2 \mathrm{H}, \mathrm{Ar}-\mathrm{H}), 7.70(s, 1 \mathrm{H},=\mathrm{C}-\mathrm{H})$, 7.45-7.40 (m, 4H), $6.82(d, 2 \mathrm{H}, \mathrm{Ar}-\mathrm{H}), 3.26-3.23(m, 4 \mathrm{H})$, $1.69-1.60(m, 6 \mathrm{H}) .{ }^{13} \mathrm{C}$ NMR (100 MHz, DMSO- $\left.d_{6}, \delta, \mathrm{ppm}\right)$; $153.13,150.03,139.68,136.87,129.41,129.27,128.88,122.65$, 114.85, 49.29, 25.41, 24.28.

Prismatic single crystals of the compounds used in X-ray diffraction studies were grown from their solutions in a acetonitrile-DMF (5:1 v:v) mixture by slow evaporation of the solvent.

\section{Refinement}

Crystal data, data collection and structure refinement details are summarized in Table 4 . $\mathrm{H}$ atoms bonded to $\mathrm{C}$ were positioned with idealized geometry and refined using a riding model with the aromatic $\mathrm{C}-\mathrm{H}=0.93,0.96$ (methyl), or $0.97 \AA$ (methylene). $\mathrm{H}$ atoms of the $\mathrm{NH}$ groups were located in a difference map and their positions refined. All $\mathrm{H}$ atoms were refined with $U_{\text {iso }}(\mathrm{H})=1.2 U_{\text {eq }}(\mathrm{C}$-aromatic, C-methylene, $\mathrm{N})$ or $1.5 U_{\text {eq }}\left(\mathrm{C}\right.$-methyl). In compound (III), the $U^{\mathrm{ij}}$ components of atoms $\mathrm{C} 14, \mathrm{C} 15, \mathrm{C} 17$, and $\mathrm{C} 18$ were restrained to approximate isotropic behaviour.

\section{Acknowledgements}

The authors thank SAIF Panjab University for extending the services of the NMR facility.

\section{Funding information}

NP thanks the Department of Science and Technology, Government of India, New Delhi, for a research fellowship 
Table 4

Experimental details.

(I)

Crystal data

Chemical formula

$M_{\mathrm{r}}$

Crystal system, space group

Temperature (K)

$a, b, c(\AA)$

$\beta\left({ }^{\circ}\right)$

$V\left(\AA^{3}\right)$

Z

Radiation type

$\mu\left(\mathrm{mm}^{-1}\right)$

Crystal size (mm)

Data collection

Diffractometer

Absorption correction

$T_{\min }, T_{\max }$

No. of measured, independent and

observed $[I>2 \sigma(I)]$ reflections

$R_{\text {int }}$

$(\sin \theta / \lambda)_{\max }\left(\AA^{-1}\right)$

$\mathrm{C}_{18} \mathrm{H}_{21} \mathrm{~N}_{3} \mathrm{O}_{2} \mathrm{~S}$

343.44

Monoclinic, $P 2_{1} / c$

293

19.221 (2), 5.4270 (7), 17.143 (2)

$105.45(2)$

$1723.6(4)$

4

Mo $K \alpha$

0.20

$0.44 \times 0.32 \times 0.28$

Oxford Diffraction Xcalibur diffractometer with Sapphire CCD

Multi-scan (CrysAlis RED; Oxford Diffraction, 2009)

$0.916,0.945$

$6052,3164,2475$

0.019

0.602

Refinement

$R\left[F^{2}>2 \sigma\left(F^{2}\right)\right], w R\left(F^{2}\right), S$

No. of reflections

No. of parameters

No. of restraints

H-atom treatment

$\Delta \rho_{\max }, \Delta \rho_{\min }\left(\mathrm{e} \AA^{-3}\right)$
(II)

$\mathrm{C}_{19} \mathrm{H}_{23} \mathrm{~N}_{3} \mathrm{O}_{2} \mathrm{~S}$
357.46
Monoclinic, $P 2_{1} / c$
293
$18.442(2), 5.3250(4), 19.412(2)$
$101.74(1)$
$1866.5(3)$
4
Mo $K \alpha$
0.19
$0.48 \times 0.24 \times 0.10$

Oxford Diffraction Xcalibur diffractometer with Sapphire CCD

Multi-scan (CrysAlis RED; Oxford Diffraction, 2009)

$0.914,0.981$

$6735,3422,2166$

0.026

0.602

$0.051,0.137,1.02$
3422
230
0
$\mathrm{H}$ atoms treated by a mixture of
$\quad$ independent and constrained
$\quad$ refinement
$0.21,-0.22$

(III)

$\mathrm{C}_{18} \mathrm{H}_{20} \mathrm{ClN}_{3} \mathrm{O}_{2} \mathrm{~S}$
377.88
Monoclinic, $\mathrm{C} 2 / c$
293
$33.052(6), 5.258(1), 43.026(8)$
$94.05(2)$
$7459(2)$
16
$\mathrm{Mo} \mathrm{K \alpha}$
0.33
$0.50 \times 0.26 \times 0.14$

Oxford Diffraction Xcalibur diffractometer with Sapphire CCD

Multi-scan (CrysAlis RED; Oxford Diffraction, 2009)

$0.851,0.955$

14002, 6833, 2999

0.042

0.602

$0.075,0.172,1.01$
6833
457
31
$\mathrm{H}$ atoms treated by a mixture of
$\quad$ independent and constrained
$\quad$ refinement
$0.27,-0.30$

Computer programs: CrysAlis CCD and CrysAlis RED (Oxford Diffraction, 2009), SHELXS2013/1 (Sheldrick, 2008), SHELXL2014/6 (Sheldrick, 2015) and PLATON (Spek, 2003).

under its PURSE Program and BTG thanks the University Grants Commission, Government of India, New Delhi, for a special grant under a UGC-BSR one-time grant to faculty.

\section{References}

Comins, D. L., Brooks, C. A. \& Ingalls, C. L. (2001). J. Org. Chem. 66, 2181-2182.

Cremer, D. \& Pople, A. J. (1975). J. Am. Chem. Soc. 97, 1354-1358.

Grishina, G. V., Gaidatova, F. L. \& Zefirov, N. S. (1995). Chem. Heterocycl. Com. 30, 1401-1426.

Gu, W., Wu, R., Qi, S., Gu, C., Si, F. \& Chen, Z. (2012). Molecules, 17, 4634-4650.

Hirshfeld, F. L. (1977). Theor. Chim. Acta, 44, 129-138.

Kang, D., Fang, Z., Huang, B., Zhang, L., Liu, U., Pannecouque, C., Naesens, L., De Clercq, E., Zhan, P. \& Liu, X. (2015). Chem. Biol. Drug Des. 86, 568-577.

Kiasalari, Z., Khalili, M., Roghani, M., Ahmadi, A. \& Mireie, M. (2014). Iran J. Pathol. 9, 138-148.

McKinnon, J. J., Jayatilaka, D. \& Spackman, M. A. (2007). Chem. Commun. 3814-3816.

Moss, G. P. (1996). Pure Appl. Chem. 68, 2193-2222.

Nallini, A., Saraboji, K., Ponnuswamy, M. N., Venkatraj, M. \& Jeyaraman, R. (2003). Mol. Cryst. Liq. Cryst. 403, 57-65.

Nardelli, M. (1983). Comput. Chem. 7, 95-98.

N'Gouan, A. J., Mansilla-Koblavi, F., Timotou, A., Adjou, A. \& Ebby, N. (2009). Acta Cryst. E65, o2880.

Oxford Diffraction (2009). CrysAlis CCD and CrysAlis RED. Oxford Diffraction Ltd., Abingdon, England.
Parekh, J., Inamdhar, P., Nair, R., Baluja, S. \& Chanda, S. (2005). J. Serb. Chem. Soc. 70, 1155-1162.

Parvez, M., Bakhtiar, M., Baqir, M. \& Zia-ur-Rehman, M. (2014). J. Chem. Crystallogr. 44, 580-585.

Purandara, H., Foro, S. \& Thimme Gowda, B. (2017). Acta Cryst. E73, 1946-1951.

Sahu, K., Behera, K., Pathaik, R. C., Nayak, A. \& Behera, G. B. (1979). Indian J. Chem. Sect. B, 18, 557-561.

Sampath, N. (2017). J. Struct. Chem. 58, 804-808.

Shah, S., Vyas, R. \& Mehta, R. H. (1992). J. Indian Chem. Soc. 69, 590-596.

Sheldrick, G. M. (2008). Acta Cryst. A64, 112-122.

Sheldrick, G. M. (2015). Acta Cryst. C71, 3-8.

Shit, S., Marschner, C. \& Mitra, S. (2016). Acta Chim. Slov. 63, 129137.

Soman, R., Sujatha, S. \& Arunkumar, C. (2014). J. Fluor. Chem. 163, 16-22.

Spackman, M. A. \& Jayatilaka, D. (2009). CrystEngComm, 11, 1932.

Spek, A. L. (2003). J. Appl. Cryst. 36, 7-13.

Tharini, K. \& Sangeetha, P. (2015). Int. J. Chem. Sci. 13, 1794 1804.

Turner, M. J., McKinnon, J. J., Wolff, S. K., Grimwood, D. J., Spackman, P. R., Jayatilaka, D. \& Spackman, M. A. (2017). CrystalExplorer17. University of Western Australia.

Wang, C. L. \& Wuorola, M. A. (1992). Org. Prep. Proceed. Int. 24, 585-621.

Yapo, Y. M., Kakou Yao, R., Timotou, A., N'Gouan, A. J. \& Tenon, A. J. (2008). Phys. Chem. News 40, 77-80. 


\section{supporting information}

Acta Cryst. (2018). E74, 1826-1832［https://doi.org/10.1107/S2056989018016237]

\section{Crystal structure and Hirshfeld surface analysis of (E)- $N^{\prime}$-[4-(piperidin-1-} yl)benzylidene]arylsulfonohydrazides

\section{Nikhila Pai, Sabine Foro and B. Thimme Gowda}

Computing details

For all structures, data collection: CrysAlis CCD (Oxford Diffraction, 2009); cell refinement: CrysAlis RED (Oxford Diffraction, 2009); data reduction: CrysAlis RED (Oxford Diffraction, 2009); program(s) used to solve structure:

SHELXS2013/1 (Sheldrick, 2008); program(s) used to refine structure: SHELXL2014/6 (Sheldrick, 2015); molecular graphics: PLATON (Spek, 2003); software used to prepare material for publication: SHELXL2014/6 (Sheldrick, 2015).

(E)-N'-[4-(Piperidin-1-yl)benzylidene]benzenesulfonohydrazide (I)

\section{Crystal data}

$\mathrm{C}_{18} \mathrm{H}_{21} \mathrm{~N}_{3} \mathrm{O}_{2} \mathrm{~S}$

$M_{r}=343.44$

Monoclinic, $P 2_{1} / c$

$a=19.221(2) \AA$

$b=5.4270(7) \AA$

$c=17.143(2) \AA$

$\beta=105.45(2)^{\circ}$

$V=1723.6(4) \AA^{3}$

$Z=4$

\section{Data collection}

Oxford Diffraction Xcalibur diffractometer with Sapphire CCD

Radiation source: Enhance (Mo) X-ray Source

Rotation method data acquisition using $\omega$ scans.

Absorption correction: multi-scan

(CrysAlis RED; Oxford Diffraction, 2009)

$T_{\text {min }}=0.916, T_{\max }=0.945$

6052 measured reflections

\section{Refinement}

Refinement on $F^{2}$

Least-squares matrix: full

$R\left[F^{2}>2 \sigma\left(F^{2}\right)\right]=0.043$

$w R\left(F^{2}\right)=0.108$

$S=1.06$

3164 reflections

220 parameters

0 restraints
$F(000)=728$

$D_{\mathrm{x}}=1.323 \mathrm{Mg} \mathrm{m}^{-3}$

Mo $K \alpha$ radiation, $\lambda=0.71073 \AA$

Cell parameters from 2039 reflections

$\theta=2.8-27.8^{\circ}$

$\mu=0.20 \mathrm{~mm}^{-1}$

$T=293 \mathrm{~K}$

Prism, colourless

$0.44 \times 0.32 \times 0.28 \mathrm{~mm}$

3164 independent reflections

2475 reflections with $I>2 \sigma(I)$

$R_{\text {int }}=0.019$

$\theta_{\max }=25.4^{\circ}, \theta_{\min }=2.8^{\circ}$

$h=-23 \rightarrow 20$

$k=-4 \rightarrow 6$

$l=-19 \rightarrow 20$

Hydrogen site location: mixed

$\mathrm{H}$ atoms treated by a mixture of independent and constrained refinement

$w=1 /\left[\sigma^{2}\left(F_{\mathrm{o}}^{2}\right)+(0.0438 P)^{2}+0.7956 P\right]$ where $P=\left(F_{\mathrm{o}}{ }^{2}+2 F_{\mathrm{c}}{ }^{2}\right) / 3$

$(\Delta / \sigma)_{\max }=0.001$

$\Delta \rho_{\max }=0.24 \mathrm{e} \AA^{-3}$

$\Delta \rho_{\min }=-0.31 \mathrm{e} \AA^{-3}$ 


\section{Special details}

Geometry. All esds (except the esd in the dihedral angle between two 1.s. planes) are estimated using the full covariance matrix. The cell esds are taken into account individually in the estimation of esds in distances, angles and torsion angles; correlations between esds in cell parameters are only used when they are defined by crystal symmetry. An approximate (isotropic) treatment of cell esds is used for estimating esds involving l.s. planes.

Fractional atomic coordinates and isotropic or equivalent isotropic displacement parameters $\left(\hat{A}^{2}\right)$

\begin{tabular}{|c|c|c|c|c|}
\hline & $x$ & $y$ & $z$ & $U_{\text {iso }} * / U_{\text {eq }}$ \\
\hline $\mathrm{C} 1$ & $0.60577(10)$ & $0.1520(4)$ & $0.46537(12)$ & $0.0384(5)$ \\
\hline $\mathrm{C} 2$ & $0.63592(13)$ & $-0.0066(5)$ & $0.42141(15)$ & $0.0553(6)$ \\
\hline $\mathrm{H} 2$ & 0.6625 & -0.1422 & 0.4460 & $0.066^{*}$ \\
\hline $\mathrm{C} 3$ & $0.62614(16)$ & $0.0387(6)$ & 0.33995 (17) & $0.0720(8)$ \\
\hline H3 & 0.6459 & -0.0684 & 0.3093 & $0.086^{*}$ \\
\hline $\mathrm{C} 4$ & $0.58782(15)$ & $0.2386(6)$ & $0.30389(15)$ & $0.0699(8)$ \\
\hline $\mathrm{H} 4$ & 0.5821 & 0.2681 & 0.2491 & $0.084^{*}$ \\
\hline $\mathrm{C} 5$ & $0.55771(13)$ & $0.3960(6)$ & $0.34822(16)$ & $0.0692(8)$ \\
\hline H5 & 0.5311 & 0.5309 & 0.3232 & $0.083^{*}$ \\
\hline C6 & $0.56663(12)$ & $0.3554(5)$ & 0.42987 (14) & $0.0551(6)$ \\
\hline H6 & 0.5467 & 0.4627 & 0.4603 & $0.066^{*}$ \\
\hline $\mathrm{C} 7$ & 0.79265 (11) & 0.4355 (4) & $0.63390(11)$ & $0.0403(5)$ \\
\hline H7 & 0.7761 & 0.5782 & 0.6531 & $0.048^{*}$ \\
\hline $\mathrm{C} 8$ & $0.86830(11)$ & $0.4209(4)$ & 0.63351 (11) & $0.0378(5)$ \\
\hline C9 & $0.89690(11)$ & $0.2255(4)$ & $0.59921(12)$ & $0.0434(5)$ \\
\hline H9 & 0.8667 & 0.0973 & 0.5749 & $0.052 *$ \\
\hline $\mathrm{C} 10$ & 0.96855 (11) & $0.2183(4)$ & $0.60049(12)$ & $0.0431(5)$ \\
\hline H10 & 0.9857 & 0.0851 & 0.5770 & $0.052^{*}$ \\
\hline C11 & $1.01676(10)$ & 0.4064 (4) & $0.63636(11)$ & $0.0353(4)$ \\
\hline $\mathrm{C} 12$ & $0.98802(11)$ & $0.5993(4)$ & $0.67192(12)$ & $0.0428(5)$ \\
\hline H12 & 1.0181 & 0.7265 & 0.6972 & $0.051^{*}$ \\
\hline $\mathrm{C} 13$ & $0.91592(12)$ & 0.6049 (4) & $0.67031(12)$ & $0.0435(5)$ \\
\hline H13 & 0.8987 & 0.7361 & 0.6947 & $0.052 *$ \\
\hline C14 & $1.10674(12)$ & $0.2969(5)$ & $0.56571(14)$ & $0.0529(6)$ \\
\hline H14A & 1.0889 & 0.4113 & 0.5214 & $0.063^{*}$ \\
\hline H14B & 1.0816 & 0.1418 & 0.5511 & $0.063^{*}$ \\
\hline C15 & $1.18625(13)$ & $0.2563(5)$ & $0.57619(18)$ & $0.0657(7)$ \\
\hline H15A & 1.2026 & 0.1212 & 0.6137 & $0.079 *$ \\
\hline H15B & 1.1941 & 0.2101 & 0.5245 & $0.079 *$ \\
\hline C16 & $1.22997(12)$ & $0.4823(5)$ & $0.60752(15)$ & $0.0558(6)$ \\
\hline H16A & 1.2810 & 0.4462 & 0.6174 & $0.067 *$ \\
\hline H16B & 1.2178 & 0.6135 & 0.5678 & $0.067^{*}$ \\
\hline C17 & $1.21388(12)$ & $0.5618(5)$ & $0.68471(14)$ & $0.0595(7)$ \\
\hline H17A & 1.2402 & 0.7120 & 0.7040 & $0.071 *$ \\
\hline H17B & 1.2302 & 0.4356 & 0.7256 & $0.071^{*}$ \\
\hline C18 & $1.13426(12)$ & $0.6062(5)$ & $0.67295(15)$ & $0.0573(6)$ \\
\hline H18A & 1.1257 & 0.6457 & 0.7248 & $0.069^{*}$ \\
\hline H18B & 1.1195 & 0.7471 & 0.6376 & $0.069^{*}$ \\
\hline N1 & $0.67909(9)$ & $0.2983(4)$ & $0.61782(11)$ & $0.0422(4)$ \\
\hline
\end{tabular}




\begin{tabular}{lllll}
$\mathrm{H} 1 \mathrm{~N}$ & $0.6650(12)$ & $0.444(4)$ & $0.6160(14)$ & $0.051^{*}$ \\
$\mathrm{~N} 2$ & $0.74846(9)$ & $0.2602(3)$ & $0.60892(10)$ & $0.0412(4)$ \\
$\mathrm{N} 3$ & $1.09002(9)$ & $0.3935(3)$ & $0.63815(9)$ & $0.0387(4)$ \\
$\mathrm{O} 1$ & $0.64512(9)$ & $-0.1391(3)$ & $0.58934(10)$ & $0.0571(4)$ \\
$\mathrm{O} 2$ & $0.55362(8)$ & $0.1803(3)$ & $0.59001(9)$ & $0.0547(4)$ \\
$\mathrm{S} 1$ & $0.61709(3)$ & $0.10285(10)$ & $0.56935(3)$ & $0.04091(17)$ \\
\hline
\end{tabular}

Atomic displacement parameters $\left(\AA^{2}\right)$

\begin{tabular}{|c|c|c|c|c|c|c|}
\hline & $U^{11}$ & $U^{22}$ & $U^{33}$ & $U^{12}$ & $U^{13}$ & $U^{23}$ \\
\hline $\mathrm{C} 1$ & $0.0281(10)$ & $0.0418(12)$ & $0.0442(11)$ & $-0.0031(9)$ & $0.0075(8)$ & $-0.0030(9)$ \\
\hline $\mathrm{C} 2$ & $0.0554(14)$ & $0.0543(14)$ & $0.0594(15)$ & $0.0026(12)$ & $0.0210(11)$ & $-0.0046(12)$ \\
\hline $\mathrm{C} 3$ & 0.0813 (19) & $0.083(2)$ & $0.0578(16)$ & $-0.0118(17)$ & $0.0297(14)$ & $-0.0194(16)$ \\
\hline $\mathrm{C} 4$ & $0.0640(16)$ & $0.102(2)$ & $0.0386(13)$ & $-0.0277(17)$ & $0.0046(12)$ & $-0.0034(15)$ \\
\hline $\mathrm{C} 5$ & $0.0536(15)$ & $0.089(2)$ & $0.0564(16)$ & $0.0059(15)$ & $-0.0001(12)$ & $0.0206(15)$ \\
\hline C6 & $0.0480(13)$ & $0.0631(16)$ & $0.0524(14)$ & $0.0131(12)$ & $0.0103(10)$ & $0.0074(12)$ \\
\hline $\mathrm{C} 7$ & $0.0473(12)$ & 0.0377 (12) & $0.0347(11)$ & $0.0034(10)$ & $0.0089(9)$ & $0.0014(9)$ \\
\hline $\mathrm{C} 8$ & $0.0426(11)$ & $0.0356(11)$ & $0.0324(10)$ & $-0.0006(9)$ & $0.0050(8)$ & $0.0045(9)$ \\
\hline C9 & $0.0443(12)$ & $0.0349(11)$ & $0.0459(12)$ & $-0.0086(10)$ & $0.0033(9)$ & $-0.0063(10)$ \\
\hline $\mathrm{C} 10$ & $0.0465(12)$ & $0.0318(11)$ & 0.0483 (12) & $-0.0028(10)$ & $0.0079(10)$ & $-0.0092(10)$ \\
\hline C11 & $0.0432(11)$ & $0.0312(10)$ & $0.0278(9)$ & $-0.0037(9)$ & $0.0027(8)$ & $0.0018(8)$ \\
\hline $\mathrm{C} 12$ & $0.0503(12)$ & 0.0339 (11) & $0.0415(11)$ & $-0.0107(10)$ & $0.0073(9)$ & $-0.0086(9)$ \\
\hline $\mathrm{C} 13$ & $0.0523(12)$ & $0.0352(11)$ & $0.0430(11)$ & $-0.0014(10)$ & $0.0126(9)$ & $-0.0063(10)$ \\
\hline C14 & $0.0505(13)$ & $0.0579(15)$ & $0.0492(13)$ & $-0.0097(11)$ & $0.0116(10)$ & $-0.0172(12)$ \\
\hline $\mathrm{C} 15$ & $0.0519(14)$ & $0.0626(17)$ & 0.0857 (19) & $-0.0081(13)$ & 0.0235 (13) & $-0.0283(15)$ \\
\hline $\mathrm{C} 16$ & $0.0453(13)$ & $0.0577(15)$ & $0.0657(15)$ & $-0.0070(12)$ & $0.0171(11)$ & $-0.0130(12)$ \\
\hline C17 & $0.0481(13)$ & $0.0739(18)$ & 0.0535 (14) & $-0.0195(13)$ & $0.0082(11)$ & $-0.0148(13)$ \\
\hline C18 & $0.0536(14)$ & $0.0606(16)$ & $0.0598(15)$ & $-0.0205(12)$ & $0.0191(11)$ & $-0.0261(12)$ \\
\hline N1 & $0.0397(10)$ & $0.0413(10)$ & $0.0444(10)$ & $0.0028(8)$ & $0.0089(8)$ & $-0.0016(9)$ \\
\hline N2 & $0.0382(9)$ & $0.0433(10)$ & $0.0393(9)$ & $0.0021(8)$ & $0.0055(7)$ & $0.0022(8)$ \\
\hline N3 & $0.0422(9)$ & $0.0387(10)$ & $0.0330(9)$ & $-0.0091(8)$ & $0.0062(7)$ & $-0.0053(7)$ \\
\hline O1 & $0.0619(10)$ & $0.0391(9)$ & $0.0689(11)$ & $0.0043(8)$ & $0.0147(8)$ & $0.0115(8)$ \\
\hline $\mathrm{O} 2$ & $0.0444(9)$ & $0.0671(11)$ & $0.0572(9)$ & $0.0011(8)$ & $0.0216(7)$ & $0.0009(8)$ \\
\hline $\mathrm{S} 1$ & $0.0378(3)$ & 0.0397 (3) & $0.0456(3)$ & $0.0016(2)$ & $0.0118(2)$ & $0.0042(2)$ \\
\hline
\end{tabular}

Geometric parameters $\left(\AA,{ }^{\circ}\right)$

\begin{tabular}{llll}
\hline $\mathrm{C} 1-\mathrm{C} 2$ & $1.370(3)$ & $\mathrm{C} 12-\mathrm{H} 12$ & 0.9300 \\
$\mathrm{C} 1-\mathrm{C} 6$ & $1.383(3)$ & $\mathrm{C} 13-\mathrm{H} 13$ & 0.9300 \\
$\mathrm{C} 1-\mathrm{S} 1$ & $1.758(2)$ & $\mathrm{C} 14-\mathrm{N} 3$ & $1.460(3)$ \\
$\mathrm{C} 2-\mathrm{C} 3$ & $1.381(4)$ & $\mathrm{C} 14-\mathrm{C} 15$ & $1.507(3)$ \\
$\mathrm{C} 2-\mathrm{H} 2$ & 0.9300 & $\mathrm{C} 14-\mathrm{H} 14 \mathrm{~A}$ & 0.9700 \\
$\mathrm{C} 3-\mathrm{C} 4$ & $1.363(4)$ & $\mathrm{C} 14-\mathrm{H} 14 \mathrm{~B}$ & 0.9700 \\
$\mathrm{C} 3-\mathrm{H} 3$ & 0.9300 & $\mathrm{C} 15-\mathrm{C} 16$ & $1.503(3)$ \\
$\mathrm{C} 4-\mathrm{C} 5$ & $1.370(4)$ & $\mathrm{C} 15-\mathrm{H} 15 \mathrm{~A}$ & 0.9700 \\
$\mathrm{C} 4-\mathrm{H} 4$ & 0.9300 & $\mathrm{C} 15-\mathrm{H} 15 \mathrm{~B}$ & 0.9700 \\
$\mathrm{C} 5-\mathrm{C} 6$ & $1.382(3)$ & $\mathrm{C} 16-\mathrm{C} 17$ & $1.500(3)$ \\
$\mathrm{C} 5-\mathrm{H} 5$ & 0.9300 & $\mathrm{C} 16-\mathrm{H} 16 \mathrm{~A}$ & 0.9700
\end{tabular}




\begin{tabular}{|c|c|c|c|}
\hline $\mathrm{C} 6-\mathrm{H} 6$ & 0.9300 & $\mathrm{C} 16-\mathrm{H} 16 \mathrm{~B}$ & 0.9700 \\
\hline $\mathrm{C} 7-\mathrm{N} 2$ & $1.271(3)$ & $\mathrm{C} 17-\mathrm{C} 18$ & $1.509(3)$ \\
\hline $\mathrm{C} 7-\mathrm{C} 8$ & $1.458(3)$ & $\mathrm{C} 17-\mathrm{H} 17 \mathrm{~A}$ & 0.9700 \\
\hline $\mathrm{C} 7-\mathrm{H} 7$ & 0.9300 & C17-H17B & 0.9700 \\
\hline $\mathrm{C} 8-\mathrm{C} 13$ & $1.387(3)$ & $\mathrm{C} 18-\mathrm{N} 3$ & $1.463(3)$ \\
\hline $\mathrm{C} 8-\mathrm{C} 9$ & $1.395(3)$ & $\mathrm{C} 18-\mathrm{H} 18 \mathrm{~A}$ & 0.9700 \\
\hline $\mathrm{C} 9-\mathrm{C} 10$ & $1.372(3)$ & $\mathrm{C} 18-\mathrm{H} 18 \mathrm{~B}$ & 0.9700 \\
\hline C9-H9 & 0.9300 & $\mathrm{~N} 1-\mathrm{N} 2$ & $1.397(2)$ \\
\hline $\mathrm{C} 10-\mathrm{C} 11$ & $1.406(3)$ & $\mathrm{N} 1-\mathrm{S} 1$ & $1.6458(19)$ \\
\hline $\mathrm{C} 10-\mathrm{H} 10$ & 0.9300 & $\mathrm{~N} 1-\mathrm{H} 1 \mathrm{~N}$ & $0.84(2)$ \\
\hline $\mathrm{C} 11-\mathrm{C} 12$ & $1.397(3)$ & $\mathrm{O} 1-\mathrm{S} 1$ & $1.4258(16)$ \\
\hline $\mathrm{C} 11-\mathrm{N} 3$ & $1.402(2)$ & $\mathrm{O} 2-\mathrm{S} 1$ & $1.4216(15)$ \\
\hline $\mathrm{C} 12-\mathrm{C} 13$ & $1.379(3)$ & & \\
\hline $\mathrm{C} 2-\mathrm{C} 1-\mathrm{C} 6$ & $121.3(2)$ & $\mathrm{C} 15-\mathrm{C} 14-\mathrm{H} 14 \mathrm{~A}$ & 108.9 \\
\hline $\mathrm{C} 2-\mathrm{C} 1-\mathrm{S} 1$ & $120.43(17)$ & $\mathrm{N} 3-\mathrm{C} 14-\mathrm{H} 14 \mathrm{~B}$ & 108.9 \\
\hline $\mathrm{C} 6-\mathrm{C} 1-\mathrm{S} 1$ & $118.24(17)$ & $\mathrm{C} 15-\mathrm{C} 14-\mathrm{H} 14 \mathrm{~B}$ & 108.9 \\
\hline $\mathrm{C} 1-\mathrm{C} 2-\mathrm{C} 3$ & $118.8(2)$ & $\mathrm{H} 14 \mathrm{~A}-\mathrm{C} 14-\mathrm{H} 14 \mathrm{~B}$ & 107.7 \\
\hline $\mathrm{C} 1-\mathrm{C} 2-\mathrm{H} 2$ & 120.6 & $\mathrm{C} 16-\mathrm{C} 15-\mathrm{C} 14$ & $112.1(2)$ \\
\hline $\mathrm{C} 3-\mathrm{C} 2-\mathrm{H} 2$ & 120.6 & $\mathrm{C} 16-\mathrm{C} 15-\mathrm{H} 15 \mathrm{~A}$ & 109.2 \\
\hline $\mathrm{C} 4-\mathrm{C} 3-\mathrm{C} 2$ & $120.8(3)$ & $\mathrm{C} 14-\mathrm{C} 15-\mathrm{H} 15 \mathrm{~A}$ & 109.2 \\
\hline $\mathrm{C} 4-\mathrm{C} 3-\mathrm{H} 3$ & 119.6 & $\mathrm{C} 16-\mathrm{C} 15-\mathrm{H} 15 \mathrm{~B}$ & 109.2 \\
\hline $\mathrm{C} 2-\mathrm{C} 3-\mathrm{H} 3$ & 119.6 & C14-C15-Н15B & 109.2 \\
\hline $\mathrm{C} 3-\mathrm{C} 4-\mathrm{C} 5$ & $120.1(2)$ & $\mathrm{H} 15 \mathrm{~A}-\mathrm{C} 15-\mathrm{H} 15 \mathrm{~B}$ & 107.9 \\
\hline $\mathrm{C} 3-\mathrm{C} 4-\mathrm{H} 4$ & 119.9 & $\mathrm{C} 17-\mathrm{C} 16-\mathrm{C} 15$ & $108.8(2)$ \\
\hline $\mathrm{C} 5-\mathrm{C} 4-\mathrm{H} 4$ & 119.9 & $\mathrm{C} 17-\mathrm{C} 16-\mathrm{H} 16 \mathrm{~A}$ & 109.9 \\
\hline $\mathrm{C} 4-\mathrm{C} 5-\mathrm{C} 6$ & $120.4(3)$ & $\mathrm{C} 15-\mathrm{C} 16-\mathrm{H} 16 \mathrm{~A}$ & 109.9 \\
\hline $\mathrm{C} 4-\mathrm{C} 5-\mathrm{H} 5$ & 119.8 & $\mathrm{C} 17-\mathrm{C} 16-\mathrm{H} 16 \mathrm{~B}$ & 109.9 \\
\hline $\mathrm{C} 6-\mathrm{C} 5-\mathrm{H} 5$ & 119.8 & $\mathrm{C} 15-\mathrm{C} 16-\mathrm{H} 16 \mathrm{~B}$ & 109.9 \\
\hline $\mathrm{C} 5-\mathrm{C} 6-\mathrm{C} 1$ & $118.6(2)$ & $\mathrm{H} 16 \mathrm{~A}-\mathrm{C} 16-\mathrm{H} 16 \mathrm{~B}$ & 108.3 \\
\hline $\mathrm{C} 5-\mathrm{C} 6-\mathrm{H} 6$ & 120.7 & $\mathrm{C} 16-\mathrm{C} 17-\mathrm{C} 18$ & $111.60(19)$ \\
\hline $\mathrm{C} 1-\mathrm{C} 6-\mathrm{H} 6$ & 120.7 & $\mathrm{C} 16-\mathrm{C} 17-\mathrm{H} 17 \mathrm{~A}$ & 109.3 \\
\hline $\mathrm{N} 2-\mathrm{C} 7-\mathrm{C} 8$ & $122.39(19)$ & $\mathrm{C} 18-\mathrm{C} 17-\mathrm{H} 17 \mathrm{~A}$ & 109.3 \\
\hline $\mathrm{N} 2-\mathrm{C} 7-\mathrm{H} 7$ & 118.8 & $\mathrm{C} 16-\mathrm{C} 17-\mathrm{H} 17 \mathrm{~B}$ & 109.3 \\
\hline $\mathrm{C} 8-\mathrm{C} 7-\mathrm{H} 7$ & 118.8 & $\mathrm{C} 18-\mathrm{C} 17-\mathrm{H} 17 \mathrm{~B}$ & 109.3 \\
\hline $\mathrm{C} 13-\mathrm{C} 8-\mathrm{C} 9$ & $116.90(19)$ & $\mathrm{H} 17 \mathrm{~A}-\mathrm{C} 17-\mathrm{H} 17 \mathrm{~B}$ & 108.0 \\
\hline $\mathrm{C} 13-\mathrm{C} 8-\mathrm{C} 7$ & $119.79(19)$ & N3- $18-\mathrm{C} 17$ & $112.8(2)$ \\
\hline $\mathrm{C} 9-\mathrm{C} 8-\mathrm{C} 7$ & $123.29(19)$ & N3-C18-H18A & 109.0 \\
\hline $\mathrm{C} 10-\mathrm{C} 9-\mathrm{C} 8$ & $121.45(19)$ & $\mathrm{C} 17-\mathrm{C} 18-\mathrm{H} 18 \mathrm{~A}$ & 109.0 \\
\hline $\mathrm{C} 10-\mathrm{C} 9-\mathrm{H} 9$ & 119.3 & $\mathrm{~N} 3-\mathrm{C} 18-\mathrm{H} 18 \mathrm{~B}$ & 109.0 \\
\hline $\mathrm{C} 8-\mathrm{C} 9-\mathrm{H} 9$ & 119.3 & $\mathrm{C} 17-\mathrm{C} 18-\mathrm{H} 18 \mathrm{~B}$ & 109.0 \\
\hline $\mathrm{C} 9-\mathrm{C} 10-\mathrm{C} 11$ & $121.9(2)$ & $\mathrm{H} 18 \mathrm{~A}-\mathrm{C} 18-\mathrm{H} 18 \mathrm{~B}$ & 107.8 \\
\hline $\mathrm{C} 9-\mathrm{C} 10-\mathrm{H} 10$ & 119.1 & $\mathrm{~N} 2-\mathrm{N} 1-\mathrm{S} 1$ & $115.68(14)$ \\
\hline $\mathrm{C} 11-\mathrm{C} 10-\mathrm{H} 10$ & 119.1 & $\mathrm{~N} 2-\mathrm{N} 1-\mathrm{H} 1 \mathrm{~N}$ & $116.4(17)$ \\
\hline $\mathrm{C} 12-\mathrm{C} 11-\mathrm{N} 3$ & $122.65(18)$ & $\mathrm{S} 1-\mathrm{N} 1-\mathrm{H} 1 \mathrm{~N}$ & $114.2(16)$ \\
\hline $\mathrm{C} 12-\mathrm{C} 11-\mathrm{C} 10$ & $116.36(19)$ & $\mathrm{C} 7-\mathrm{N} 2-\mathrm{N} 1$ & $115.09(18)$ \\
\hline $\mathrm{N} 3-\mathrm{C} 11-\mathrm{C} 10$ & $120.96(18)$ & $\mathrm{C} 11-\mathrm{N} 3-\mathrm{C} 14$ & $116.53(15)$ \\
\hline $\mathrm{C} 13-\mathrm{C} 12-\mathrm{C} 11$ & $121.31(19)$ & $\mathrm{C} 11-\mathrm{N} 3-\mathrm{C} 18$ & $116.14(17)$ \\
\hline
\end{tabular}




\begin{tabular}{|c|c|c|c|}
\hline $\mathrm{C} 13-\mathrm{C} 12-\mathrm{H} 12$ & 119.3 & $\mathrm{C} 14-\mathrm{N} 3-\mathrm{C} 18$ & $113.21(17)$ \\
\hline $\mathrm{C} 11-\mathrm{C} 12-\mathrm{H} 12$ & 119.3 & $\mathrm{O} 2-\mathrm{S} 1-\mathrm{O} 1$ & $120.46(10)$ \\
\hline $\mathrm{C} 12-\mathrm{C} 13-\mathrm{C} 8$ & $122.1(2)$ & $\mathrm{O} 2-\mathrm{S} 1-\mathrm{N} 1$ & $103.75(10)$ \\
\hline $\mathrm{C} 12-\mathrm{C} 13-\mathrm{H} 13$ & 119.0 & $\mathrm{O} 1-\mathrm{S} 1-\mathrm{N} 1$ & $107.20(10)$ \\
\hline $\mathrm{C} 8-\mathrm{C} 13-\mathrm{H} 13$ & 119.0 & $\mathrm{O} 2-\mathrm{S} 1-\mathrm{C} 1$ & $108.99(9)$ \\
\hline $\mathrm{N} 3-\mathrm{C} 14-\mathrm{C} 15$ & $113.40(18)$ & $\mathrm{O} 1-\mathrm{S} 1-\mathrm{C} 1$ & $108.67(10)$ \\
\hline $\mathrm{N} 3-\mathrm{C} 14-\mathrm{H} 14 \mathrm{~A}$ & 108.9 & $\mathrm{~N} 1-\mathrm{S} 1-\mathrm{C} 1$ & $106.95(9)$ \\
\hline $\mathrm{C} 6-\mathrm{C} 1-\mathrm{C} 2-\mathrm{C} 3$ & $-0.5(3)$ & $\mathrm{C} 15-\mathrm{C} 16-\mathrm{C} 17-\mathrm{C} 18$ & $-56.4(3)$ \\
\hline $\mathrm{S} 1-\mathrm{C} 1-\mathrm{C} 2-\mathrm{C} 3$ & $-179.69(19)$ & $\mathrm{C} 16-\mathrm{C} 17-\mathrm{C} 18-\mathrm{N} 3$ & $54.7(3)$ \\
\hline $\mathrm{C} 1-\mathrm{C} 2-\mathrm{C} 3-\mathrm{C} 4$ & $0.6(4)$ & $\mathrm{C} 8-\mathrm{C} 7-\mathrm{N} 2-\mathrm{N} 1$ & $176.43(17)$ \\
\hline $\mathrm{C} 2-\mathrm{C} 3-\mathrm{C} 4-\mathrm{C} 5$ & $-0.8(4)$ & $\mathrm{S} 1-\mathrm{N} 1-\mathrm{N} 2-\mathrm{C} 7$ & $167.78(14)$ \\
\hline $\mathrm{C} 3-\mathrm{C} 4-\mathrm{C} 5-\mathrm{C} 6$ & $0.8(4)$ & $\mathrm{C} 12-\mathrm{C} 11-\mathrm{N} 3-\mathrm{C} 14$ & $-142.9(2)$ \\
\hline $\mathrm{C} 4-\mathrm{C} 5-\mathrm{C} 6-\mathrm{C} 1$ & $-0.7(4)$ & $\mathrm{C} 10-\mathrm{C} 11-\mathrm{N} 3-\mathrm{C} 14$ & $39.3(3)$ \\
\hline $\mathrm{C} 2-\mathrm{C} 1-\mathrm{C} 6-\mathrm{C} 5$ & $0.5(3)$ & $\mathrm{C} 12-\mathrm{C} 11-\mathrm{N} 3-\mathrm{C} 18$ & $-5.6(3)$ \\
\hline $\mathrm{S} 1-\mathrm{C} 1-\mathrm{C} 6-\mathrm{C} 5$ & $179.75(18)$ & $\mathrm{C} 10-\mathrm{C} 11-\mathrm{N} 3-\mathrm{C} 18$ & $176.68(19)$ \\
\hline $\mathrm{N} 2-\mathrm{C} 7-\mathrm{C} 8-\mathrm{C} 13$ & $-172.29(19)$ & $\mathrm{C} 15-\mathrm{C} 14-\mathrm{N} 3-\mathrm{C} 11$ & $-172.2(2)$ \\
\hline $\mathrm{N} 2-\mathrm{C} 7-\mathrm{C} 8-\mathrm{C} 9$ & $6.1(3)$ & $\mathrm{C} 15-\mathrm{C} 14-\mathrm{N} 3-\mathrm{C} 18$ & $49.3(3)$ \\
\hline $\mathrm{C} 13-\mathrm{C} 8-\mathrm{C} 9-\mathrm{C} 10$ & $-1.2(3)$ & $\mathrm{C} 17-\mathrm{C} 18-\mathrm{N} 3-\mathrm{C} 11$ & $170.97(18)$ \\
\hline $\mathrm{C} 7-\mathrm{C} 8-\mathrm{C} 9-\mathrm{C} 10$ & $-179.67(19)$ & $\mathrm{C} 17-\mathrm{C} 18-\mathrm{N} 3-\mathrm{C} 14$ & $-50.3(3)$ \\
\hline $\mathrm{C} 8-\mathrm{C} 9-\mathrm{C} 10-\mathrm{C} 11$ & $0.1(3)$ & $\mathrm{N} 2-\mathrm{N} 1-\mathrm{S} 1-\mathrm{O} 2$ & $178.88(14)$ \\
\hline $\mathrm{C} 9-\mathrm{C} 10-\mathrm{C} 11-\mathrm{C} 12$ & $1.1(3)$ & $\mathrm{N} 2-\mathrm{N} 1-\mathrm{S} 1-\mathrm{O} 1$ & $50.42(17)$ \\
\hline $\mathrm{C} 9-\mathrm{C} 10-\mathrm{C} 11-\mathrm{N} 3$ & $178.96(18)$ & $\mathrm{N} 2-\mathrm{N} 1-\mathrm{S} 1-\mathrm{C} 1$ & $-65.99(16)$ \\
\hline $\mathrm{N} 3-\mathrm{C} 11-\mathrm{C} 12-\mathrm{C} 13$ & $-178.92(18)$ & $\mathrm{C} 2-\mathrm{C} 1-\mathrm{S} 1-\mathrm{O} 2$ & $-145.87(18)$ \\
\hline $\mathrm{C} 10-\mathrm{C} 11-\mathrm{C} 12-\mathrm{C} 13$ & $-1.1(3)$ & $\mathrm{C} 6-\mathrm{C} 1-\mathrm{S} 1-\mathrm{O} 2$ & $34.91(19)$ \\
\hline $\mathrm{C} 11-\mathrm{C} 12-\mathrm{C} 13-\mathrm{C} 8$ & $-0.1(3)$ & $\mathrm{C} 2-\mathrm{C} 1-\mathrm{S} 1-\mathrm{O} 1$ & $-12.9(2)$ \\
\hline $\mathrm{C} 9-\mathrm{C} 8-\mathrm{C} 13-\mathrm{C} 12$ & $1.2(3)$ & $\mathrm{C} 6-\mathrm{C} 1-\mathrm{S} 1-\mathrm{O} 1$ & $167.93(16)$ \\
\hline $\mathrm{C} 7-\mathrm{C} 8-\mathrm{C} 13-\mathrm{C} 12$ & $179.73(19)$ & $\mathrm{C} 2-\mathrm{C} 1-\mathrm{S} 1-\mathrm{N} 1$ & $102.56(19)$ \\
\hline $\mathrm{N} 3-\mathrm{C} 14-\mathrm{C} 15-\mathrm{C} 16$ & $-52.5(3)$ & $\mathrm{C} 6-\mathrm{C} 1-\mathrm{S} 1-\mathrm{N} 1$ & $-76.65(18)$ \\
\hline $\mathrm{C} 14-\mathrm{C} 15-\mathrm{C} 16-\mathrm{C} 17$ & $55.3(3)$ & & \\
\hline
\end{tabular}

Hydrogen-bond geometry $\left(A,{ }^{\circ}\right)$

\begin{tabular}{lllll}
\hline$D-\mathrm{H} \cdots A$ & $D-\mathrm{H}$ & $\mathrm{H} \cdots A$ & $D \cdots A$ & $D-\mathrm{H} \cdots A$ \\
\hline $\mathrm{N} 1-\mathrm{H} 1 N \cdots \mathrm{O} 1^{\mathrm{i}}$ & $0.84(2)$ & $2.32(2)$ & $3.133(3)$ & $165(2)$ \\
\hline
\end{tabular}

Symmetry code: (i) $x, y+1, z$.

(E)-4-Methyl-N'-[4-(piperidin-1-yl)benzylidene]benzenesulfonohydrazide (II)

\section{Crystal data}

$\mathrm{C}_{19} \mathrm{H}_{23} \mathrm{~N}_{3} \mathrm{O}_{2} \mathrm{~S}$

$M_{r}=357.46$

Monoclinic, $P 2_{1} / c$

$a=18.442(2) \AA$

$b=5.3250(4) \AA$

$c=19.412(2) \AA$

$\beta=101.74(1)^{\circ}$

$V=1866.5(3) \AA^{3}$

$Z=4$
$F(000)=760$

$D_{\mathrm{x}}=1.272 \mathrm{Mg} \mathrm{m}^{-3}$

Mo $K \alpha$ radiation, $\lambda=0.71073 \AA$

Cell parameters from 1748 reflections

$\theta=2.6-28.0^{\circ}$

$\mu=0.19 \mathrm{~mm}^{-1}$

$T=293 \mathrm{~K}$

Prism, light pink

$0.48 \times 0.24 \times 0.10 \mathrm{~mm}$ 


\section{Data collection}

Oxford Diffraction Xcalibur

diffractometer with Sapphire CCD

Radiation source: Enhance (Mo) X-ray Source

Rotation method data acquisition using $\omega$ scans.

Absorption correction: multi-scan

(CrysAlis RED; Oxford Diffraction, 2009)

$T_{\min }=0.914, T_{\max }=0.981$

6735 measured reflections

\section{Refinement}

Refinement on $F^{2}$

Least-squares matrix: full

$R\left[F^{2}>2 \sigma\left(F^{2}\right)\right]=0.051$

$w R\left(F^{2}\right)=0.137$

$S=1.02$

3422 reflections

230 parameters

0 restraints
3422 independent reflections

2166 reflections with $I>2 \sigma(I)$

$R_{\text {int }}=0.026$

$\theta_{\text {max }}=25.4^{\circ}, \theta_{\text {min }}=2.6^{\circ}$

$h=-22 \rightarrow 13$

$k=-6 \rightarrow 2$

$l=-21 \rightarrow 23$

Hydrogen site location: mixed

$\mathrm{H}$ atoms treated by a mixture of independent and constrained refinement

$w=1 /\left[\sigma^{2}\left(F_{\mathrm{o}}^{2}\right)+(0.0561 P)^{2}+0.7083 P\right]$

where $P=\left(F_{\mathrm{o}}{ }^{2}+2 F_{\mathrm{c}}{ }^{2}\right) / 3$

$(\Delta / \sigma)_{\max }<0.001$

$\Delta \rho_{\max }=0.21 \mathrm{e} \AA^{-3}$

$\Delta \rho_{\min }=-0.22$ e $\AA^{-3}$

Special details

Geometry. All esds (except the esd in the dihedral angle between two 1.s. planes) are estimated using the full covariance matrix. The cell esds are taken into account individually in the estimation of esds in distances, angles and torsion angles; correlations between esds in cell parameters are only used when they are defined by crystal symmetry. An approximate (isotropic) treatment of cell esds is used for estimating esds involving l.s. planes.

Fractional atomic coordinates and isotropic or equivalent isotropic displacement parameters $\left(\AA^{2}\right)$

\begin{tabular}{|c|c|c|c|c|}
\hline & $x$ & $y$ & $z$ & $U_{\text {iso }} * / U_{\text {eq }}$ \\
\hline $\mathrm{C} 1$ & 0.87009 (13) & $0.2262(5)$ & $0.74497(13)$ & $0.0464(6)$ \\
\hline $\mathrm{C} 2$ & $0.85130(16)$ & $0.0568(6)$ & $0.69097(15)$ & $0.0610(8)$ \\
\hline $\mathrm{H} 2$ & 0.8189 & -0.0742 & 0.6944 & $0.073 *$ \\
\hline $\mathrm{C} 3$ & $0.88094(18)$ & $0.0827(6)$ & $0.63160(16)$ & $0.0716(9)$ \\
\hline H3 & 0.8682 & -0.0331 & 0.5953 & $0.086^{*}$ \\
\hline $\mathrm{C} 4$ & $0.92855(15)$ & $0.2738(6)$ & $0.62451(15)$ & $0.0640(8)$ \\
\hline $\mathrm{C} 5$ & $0.94574(15)$ & $0.4437(6)$ & $0.67864(16)$ & $0.0635(8)$ \\
\hline H5 & 0.9775 & 0.5760 & 0.6746 & $0.076^{*}$ \\
\hline C6 & $0.91734(15)$ & $0.4236(5)$ & $0.73839(15)$ & $0.0579(7)$ \\
\hline H6 & 0.9296 & 0.5414 & 0.7742 & $0.070^{*}$ \\
\hline $\mathrm{C} 7$ & $0.65696(15)$ & $0.5049(5)$ & $0.75489(14)$ & $0.0549(7)$ \\
\hline $\mathrm{H} 7$ & 0.6648 & 0.6537 & 0.7804 & $0.066^{*}$ \\
\hline $\mathrm{C} 8$ & $0.58859(14)$ & $0.4736(5)$ & $0.70327(14)$ & $0.0497(7)$ \\
\hline $\mathrm{C} 9$ & $0.57536(16)$ & $0.2675(5)$ & $0.65812(16)$ & $0.0606(8)$ \\
\hline H9 & 0.6120 & 0.1463 & 0.6600 & $0.073 *$ \\
\hline $\mathrm{C} 10$ & $0.50985(16)$ & $0.2394(5)$ & $0.61123(16)$ & $0.0603(8)$ \\
\hline $\mathrm{H} 10$ & 0.5032 & 0.0999 & 0.5818 & $0.072 *$ \\
\hline $\mathrm{C} 11$ & $0.45234(15)$ & $0.4149(5)$ & $0.60617(14)$ & $0.0508(7)$ \\
\hline $\mathrm{C} 12$ & $0.46593(15)$ & $0.6186(5)$ & $0.65168(15)$ & $0.0570(7)$ \\
\hline $\mathrm{H} 12$ & 0.4293 & 0.7396 & 0.6503 & $0.068 *$ \\
\hline $\mathrm{C} 13$ & $0.53232(15)$ & $0.6458(5)$ & $0.69881(15)$ & $0.0567(7)$ \\
\hline H13 & 0.5392 & 0.7848 & 0.7284 & $0.068 *$ \\
\hline
\end{tabular}




$\begin{array}{lllll}\text { C14 } & 0.38983(18) & 0.2897(8) & 0.48900(18) & 0.0869(11) \\ \text { H14A } & 0.4194 & 0.1377 & 0.4942 & 0.104^{*} \\ \text { H14B } & 0.4156 & 0.4144 & 0.4665 & 0.104^{*} \\ \text { C15 } & 0.3168(2) & 0.2351(7) & 0.44174(19) & 0.097^{*}(12) \\ \text { H15A } & 0.3246 & 0.2074 & 0.3944 & 0.114^{*} \\ \text { H15B } & 0.2970 & 0.0811 & 0.4573 & 0.114^{*} \\ \text { C16 } & 0.2620(2) & 0.4358(7) & 0.43997(19) & 0.0892(11) \\ \text { H16A } & 0.2143 & 0.3791 & 0.4139 & 0.107^{*} \\ \text { H16B } & 0.2767 & 0.5809 & 0.4159 & 0.107^{*} \\ \text { C17 } & 0.25546(19) & 0.5082(9) & 0.5121(2) & 0.1010(13) \\ \text { H17A } & 0.2317 & 0.3727 & 0.5326 & 0.121^{*} \\ \text { H17B } & 0.2239 & 0.6550 & 0.5095 & 0.121^{*} \\ \text { C18 } & 0.32853(18) & 0.5650(8) & 0.55932(19) & 0.0931(12) \\ \text { H18A } & 0.3460 & 0.7256 & 0.5456 & 0.112^{*} \\ \text { H18B } & 0.3210 & 0.5817 & 0.6071 & 0.112^{*} \\ \text { C19 } & 0.9621(2) & 0.2953(9) & 0.56057(18) & 0.1048(13) \\ \text { H19A } & 1.0151 & 0.2897 & 0.5744 & 0.157^{*} \\ \text { H19B } & 0.9474 & 0.4515 & 0.5372 & 0.157^{*} \\ \text { H19C } & 0.9453 & 0.1585 & 0.5292 & 0.157^{*} \\ \text { N1 } & 0.76763(13) & 0.3858(4) & 0.81973(13) & 0.0564(6) \\ \text { H1N } & 0.7786(17) & 0.529(6) & 0.8265(15) & 0.068^{*} \\ \text { N2 } & 0.70639(12) & 0.3358(4) & 0.76625(12) & 0.0541(6) \\ \text { N3 } & 0.38501(12) & 0.3803(4) & 0.55849(12) & 0.0592(6) \\ \text { O1 } & 0.89223(11) & 0.2837(4) & 0.87969(10) & 0.0729(6) \\ \text { O2 } & 0.80891(11) & -0.0554(4) & 0.82456(11) & 0.0711(6) \\ \text { S1 } & 0.83734(4) & 0.19279(14) & 0.82295(4) & 0.0548(2) \\ & & & & \end{array}$

Atomic displacement parameters $\left(\AA^{2}\right)$

\begin{tabular}{lllllll}
\hline & $U^{11}$ & $U^{22}$ & $U^{33}$ & $U^{12}$ & $U^{13}$ & $U^{23}$ \\
\hline C1 & $0.0376(13)$ & $0.0478(15)$ & $0.0508(15)$ & $0.0021(12)$ & $0.0023(11)$ & $0.0067(13)$ \\
C2 & $0.0635(18)$ & $0.0558(18)$ & $0.0625(19)$ & $-0.0139(15)$ & $0.0102(15)$ & $-0.0011(16)$ \\
C3 & $0.078(2)$ & $0.075(2)$ & $0.0595(19)$ & $-0.0078(19)$ & $0.0097(16)$ & $-0.0107(17)$ \\
C4 & $0.0498(17)$ & $0.081(2)$ & $0.0595(19)$ & $0.0021(17)$ & $0.0074(14)$ & $0.0110(17)$ \\
C5 & $0.0476(16)$ & $0.073(2)$ & $0.069(2)$ & $-0.0149(15)$ & $0.0094(15)$ & $0.0132(17)$ \\
C6 & $0.0514(16)$ & $0.0578(17)$ & $0.0609(18)$ & $-0.0082(14)$ & $0.0027(14)$ & $-0.0007(15)$ \\
C7 & $0.0570(17)$ & $0.0475(16)$ & $0.0638(18)$ & $-0.0020(15)$ & $0.0205(14)$ & $0.0029(14)$ \\
C8 & $0.0489(16)$ & $0.0415(15)$ & $0.0630(17)$ & $0.0007(13)$ & $0.0212(13)$ & $0.0071(13)$ \\
C9 & $0.0555(18)$ & $0.0483(18)$ & $0.081(2)$ & $0.0126(14)$ & $0.0214(15)$ & $-0.0012(15)$ \\
C10 & $0.0601(18)$ & $0.0470(17)$ & $0.075(2)$ & $0.0066(14)$ & $0.0166(15)$ & $-0.0100(14)$ \\
C11 & $0.0536(16)$ & $0.0468(16)$ & $0.0575(17)$ & $0.0041(13)$ & $0.0244(14)$ & $0.0025(14)$ \\
C12 & $0.0540(17)$ & $0.0523(17)$ & $0.0678(18)$ & $0.0154(14)$ & $0.0196(15)$ & $-0.0018(14)$ \\
C13 & $0.0601(18)$ & $0.0447(16)$ & $0.0673(19)$ & $0.0057(14)$ & $0.0178(15)$ & $-0.0046(14)$ \\
C14 & $0.075(2)$ & $0.106(3)$ & $0.080(2)$ & $0.018(2)$ & $0.0142(18)$ & $-0.019(2)$ \\
C15 & $0.094(3)$ & $0.100(3)$ & $0.082(3)$ & $0.014(2)$ & $-0.003(2)$ & $-0.026(2)$ \\
C16 & $0.080(2)$ & $0.087(3)$ & $0.092(3)$ & $0.008(2)$ & $-0.004(2)$ & $-0.012(2)$ \\
C17 & $0.062(2)$ & $0.146(4)$ & $0.092(3)$ & $0.024(2)$ & $0.0066(19)$ & $-0.027(3)$ \\
C18 & $0.062(2)$ & $0.120(3)$ & $0.091(3)$ & $0.027(2)$ & $0.0031(18)$ & $-0.037(2)$
\end{tabular}




\begin{tabular}{lllllll}
$\mathrm{C} 19$ & $0.096(3)$ & $0.157(4)$ & $0.068(2)$ & $-0.011(3)$ & $0.034(2)$ & $0.010(2)$ \\
$\mathrm{N} 1$ & $0.0554(15)$ & $0.0502(14)$ & $0.0646(15)$ & $-0.0005(12)$ & $0.0141(12)$ & $0.0008(13)$ \\
$\mathrm{N} 2$ & $0.0468(13)$ & $0.0513(14)$ & $0.0650(15)$ & $-0.0022(12)$ & $0.0133(11)$ & $0.0058(12)$ \\
$\mathrm{N} 3$ & $0.0522(14)$ & $0.0670(16)$ & $0.0603(15)$ & $0.0086(12)$ & $0.0161(11)$ & $-0.0056(12)$ \\
$\mathrm{O} 1$ & $0.0673(13)$ & $0.0926(16)$ & $0.0530(12)$ & $0.0007(12)$ & $-0.0013(10)$ & $0.0053(11)$ \\
$\mathrm{O} 2$ & $0.0852(15)$ & $0.0493(12)$ & $0.0840(15)$ & $-0.0002(11)$ & $0.0293(12)$ & $0.0194(10)$ \\
$\mathrm{S} 1$ & $0.0529(4)$ & $0.0546(4)$ & $0.0558(4)$ & $0.0012(4)$ & $0.0083(3)$ & $0.0101(4)$ \\
\hline
\end{tabular}

Geometric parameters $\left(\AA,{ }^{\circ}\right)$

\begin{tabular}{|c|c|c|c|}
\hline $\mathrm{C} 1-\mathrm{C} 2$ & $1.373(4)$ & $\mathrm{C} 13-\mathrm{H} 13$ & 0.9300 \\
\hline $\mathrm{C} 1-\mathrm{C} 6$ & $1.388(4)$ & $\mathrm{C} 14-\mathrm{N} 3$ & $1.452(4)$ \\
\hline $\mathrm{C} 1-\mathrm{S} 1$ & $1.749(3)$ & $\mathrm{C} 14-\mathrm{C} 15$ & $1.496(4)$ \\
\hline $\mathrm{C} 2-\mathrm{C} 3$ & $1.379(4)$ & $\mathrm{C} 14-\mathrm{H} 14 \mathrm{~A}$ & 0.9700 \\
\hline $\mathrm{C} 2-\mathrm{H} 2$ & 0.9300 & $\mathrm{C} 14-\mathrm{H} 14 \mathrm{~B}$ & 0.9700 \\
\hline $\mathrm{C} 3-\mathrm{C} 4$ & $1.369(4)$ & $\mathrm{C} 15-\mathrm{C} 16$ & $1.466(5)$ \\
\hline $\mathrm{C} 3-\mathrm{H} 3$ & 0.9300 & $\mathrm{C} 15-\mathrm{H} 15 \mathrm{~A}$ & 0.9700 \\
\hline $\mathrm{C} 4-\mathrm{C} 5$ & $1.374(4)$ & $\mathrm{C} 15-\mathrm{H} 15 \mathrm{~B}$ & 0.9700 \\
\hline $\mathrm{C} 4-\mathrm{C} 19$ & $1.499(4)$ & $\mathrm{C} 16-\mathrm{C} 17$ & $1.481(5)$ \\
\hline $\mathrm{C} 5-\mathrm{C} 6$ & $1.370(4)$ & $\mathrm{C} 16-\mathrm{H} 16 \mathrm{~A}$ & 0.9700 \\
\hline $\mathrm{C} 5-\mathrm{H} 5$ & 0.9300 & $\mathrm{C} 16-\mathrm{H} 16 \mathrm{~B}$ & 0.9700 \\
\hline $\mathrm{C} 6-\mathrm{H} 6$ & 0.9300 & $\mathrm{C} 17-\mathrm{C} 18$ & $1.498(4)$ \\
\hline $\mathrm{C} 7-\mathrm{N} 2$ & $1.269(3)$ & C17-H17A & 0.9700 \\
\hline $\mathrm{C} 7-\mathrm{C} 8$ & $1.452(4)$ & C17-H17B & 0.9700 \\
\hline $\mathrm{C} 7-\mathrm{H} 7$ & 0.9300 & $\mathrm{C} 18-\mathrm{N} 3$ & $1.435(4)$ \\
\hline $\mathrm{C} 8-\mathrm{C} 13$ & $1.374(4)$ & $\mathrm{C} 18-\mathrm{H} 18 \mathrm{~A}$ & 0.9700 \\
\hline $\mathrm{C} 8-\mathrm{C} 9$ & $1.395(4)$ & $\mathrm{C} 18-\mathrm{H} 18 \mathrm{~B}$ & 0.9700 \\
\hline $\mathrm{C} 9-\mathrm{C} 10$ & $1.365(4)$ & C19-H19A & 0.9600 \\
\hline $\mathrm{C} 9-\mathrm{H} 9$ & 0.9300 & C19-H19B & 0.9600 \\
\hline $\mathrm{C} 10-\mathrm{C} 11$ & $1.402(4)$ & $\mathrm{C} 19-\mathrm{H} 19 \mathrm{C}$ & 0.9600 \\
\hline $\mathrm{C} 10-\mathrm{H} 10$ & 0.9300 & $\mathrm{~N} 1-\mathrm{N} 2$ & $1.395(3)$ \\
\hline $\mathrm{C} 11-\mathrm{C} 12$ & $1.389(4)$ & $\mathrm{N} 1-\mathrm{S} 1$ & $1.637(3)$ \\
\hline $\mathrm{C} 11-\mathrm{N} 3$ & $1.402(3)$ & $\mathrm{N} 1-\mathrm{H} 1 \mathrm{~N}$ & $0.79(3)$ \\
\hline $\mathrm{C} 12-\mathrm{C} 13$ & $1.379(4)$ & $\mathrm{O} 1-\mathrm{S} 1$ & $1.421(2)$ \\
\hline $\mathrm{C} 12-\mathrm{H} 12$ & 0.9300 & $\mathrm{O} 2-\mathrm{S} 1$ & $1.424(2)$ \\
\hline $\mathrm{C} 2-\mathrm{C} 1-\mathrm{C} 6$ & $119.5(3)$ & $\mathrm{H} 14 \mathrm{~A}-\mathrm{C} 14-\mathrm{H} 14 \mathrm{~B}$ & 107.6 \\
\hline $\mathrm{C} 2-\mathrm{C} 1-\mathrm{S} 1$ & $121.1(2)$ & $\mathrm{C} 16-\mathrm{C} 15-\mathrm{C} 14$ & $113.7(3)$ \\
\hline $\mathrm{C} 6-\mathrm{C} 1-\mathrm{S} 1$ & $119.4(2)$ & $\mathrm{C} 16-\mathrm{C} 15-\mathrm{H} 15 \mathrm{~A}$ & 108.8 \\
\hline $\mathrm{C} 1-\mathrm{C} 2-\mathrm{C} 3$ & $119.4(3)$ & $\mathrm{C} 14-\mathrm{C} 15-\mathrm{H} 15 \mathrm{~A}$ & 108.8 \\
\hline $\mathrm{C} 1-\mathrm{C} 2-\mathrm{H} 2$ & 120.3 & $\mathrm{C} 16-\mathrm{C} 15-\mathrm{H} 15 \mathrm{~B}$ & 108.8 \\
\hline $\mathrm{C} 3-\mathrm{C} 2-\mathrm{H} 2$ & 120.3 & $\mathrm{C} 14-\mathrm{C} 15-\mathrm{H} 15 \mathrm{~B}$ & 108.8 \\
\hline $\mathrm{C} 4-\mathrm{C} 3-\mathrm{C} 2$ & $122.0(3)$ & $\mathrm{H} 15 \mathrm{~A}-\mathrm{C} 15-\mathrm{H} 15 \mathrm{~B}$ & 107.7 \\
\hline $\mathrm{C} 4-\mathrm{C} 3-\mathrm{H} 3$ & 119.0 & $\mathrm{C} 15-\mathrm{C} 16-\mathrm{C} 17$ & $110.9(3)$ \\
\hline $\mathrm{C} 2-\mathrm{C} 3-\mathrm{H} 3$ & 119.0 & $\mathrm{C} 15-\mathrm{C} 16-\mathrm{H} 16 \mathrm{~A}$ & 109.5 \\
\hline $\mathrm{C} 3-\mathrm{C} 4-\mathrm{C} 5$ & $117.7(3)$ & $\mathrm{C} 17-\mathrm{C} 16-\mathrm{H} 16 \mathrm{~A}$ & 109.5 \\
\hline $\mathrm{C} 3-\mathrm{C} 4-\mathrm{C} 19$ & $121.4(3)$ & $\mathrm{C} 15-\mathrm{C} 16-\mathrm{H} 16 \mathrm{~B}$ & 109.5 \\
\hline $\mathrm{C} 5-\mathrm{C} 4-\mathrm{C} 19$ & $120.9(3)$ & $\mathrm{C} 17-\mathrm{C} 16-\mathrm{H} 16 \mathrm{~B}$ & 109.5 \\
\hline
\end{tabular}




\begin{tabular}{|c|c|c|c|}
\hline $\mathrm{C} 6-\mathrm{C} 5-\mathrm{C} 4$ & $121.9(3)$ & $\mathrm{H} 16 \mathrm{~A}-\mathrm{C} 16-\mathrm{H} 16 \mathrm{~B}$ & 108.1 \\
\hline $\mathrm{C} 6-\mathrm{C} 5-\mathrm{H} 5$ & 119.1 & $\mathrm{C} 16-\mathrm{C} 17-\mathrm{C} 18$ & $113.2(3)$ \\
\hline $\mathrm{C} 4-\mathrm{C} 5-\mathrm{H} 5$ & 119.1 & $\mathrm{C} 16-\mathrm{C} 17-\mathrm{H} 17 \mathrm{~A}$ & 108.9 \\
\hline $\mathrm{C} 5-\mathrm{C} 6-\mathrm{C} 1$ & $119.5(3)$ & $\mathrm{C} 18-\mathrm{C} 17-\mathrm{H} 17 \mathrm{~A}$ & 108.9 \\
\hline $\mathrm{C} 5-\mathrm{C} 6-\mathrm{H} 6$ & 120.3 & $\mathrm{C} 16-\mathrm{C} 17-\mathrm{H} 17 \mathrm{~B}$ & 108.9 \\
\hline $\mathrm{C} 1-\mathrm{C} 6-\mathrm{H} 6$ & 120.3 & $\mathrm{C} 18-\mathrm{C} 17-\mathrm{H} 17 \mathrm{~B}$ & 108.9 \\
\hline $\mathrm{N} 2-\mathrm{C} 7-\mathrm{C} 8$ & $122.1(3)$ & $\mathrm{H} 17 \mathrm{~A}-\mathrm{C} 17-\mathrm{H} 17 \mathrm{~B}$ & 107.7 \\
\hline $\mathrm{N} 2-\mathrm{C} 7-\mathrm{H} 7$ & 119.0 & $\mathrm{~N} 3-\mathrm{C} 18-\mathrm{C} 17$ & $114.8(3)$ \\
\hline $\mathrm{C} 8-\mathrm{C} 7-\mathrm{H} 7$ & 119.0 & $\mathrm{~N} 3-\mathrm{C} 18-\mathrm{H} 18 \mathrm{~A}$ & 108.6 \\
\hline $\mathrm{C} 13-\mathrm{C} 8-\mathrm{C} 9$ & $116.9(3)$ & $\mathrm{C} 17-\mathrm{C} 18-\mathrm{H} 18 \mathrm{~A}$ & 108.6 \\
\hline $\mathrm{C} 13-\mathrm{C} 8-\mathrm{C} 7$ & $120.4(3)$ & $\mathrm{N} 3-\mathrm{C} 18-\mathrm{H} 18 \mathrm{~B}$ & 108.6 \\
\hline $\mathrm{C} 9-\mathrm{C} 8-\mathrm{C} 7$ & $122.7(2)$ & $\mathrm{C} 17-\mathrm{C} 18-\mathrm{H} 18 \mathrm{~B}$ & 108.6 \\
\hline $\mathrm{C} 10-\mathrm{C} 9-\mathrm{C} 8$ & $121.5(3)$ & $\mathrm{H} 18 \mathrm{~A}-\mathrm{C} 18-\mathrm{H} 18 \mathrm{~B}$ & 107.5 \\
\hline $\mathrm{C} 10-\mathrm{C} 9-\mathrm{H} 9$ & 119.2 & $\mathrm{C} 4-\mathrm{C} 19-\mathrm{H} 19 \mathrm{~A}$ & 109.5 \\
\hline $\mathrm{C} 8-\mathrm{C} 9-\mathrm{H} 9$ & 119.2 & $\mathrm{C} 4-\mathrm{C} 19-\mathrm{H} 19 \mathrm{~B}$ & 109.5 \\
\hline $\mathrm{C} 9-\mathrm{C} 10-\mathrm{C} 11$ & $121.9(3)$ & $\mathrm{H} 19 \mathrm{~A}-\mathrm{C} 19-\mathrm{H} 19 \mathrm{~B}$ & 109.5 \\
\hline $\mathrm{C} 9-\mathrm{C} 10-\mathrm{H} 10$ & 119.1 & $\mathrm{C} 4-\mathrm{C} 19-\mathrm{H} 19 \mathrm{C}$ & 109.5 \\
\hline $\mathrm{C} 11-\mathrm{C} 10-\mathrm{H} 10$ & 119.1 & $\mathrm{H} 19 \mathrm{~A}-\mathrm{C} 19-\mathrm{H} 19 \mathrm{C}$ & 109.5 \\
\hline $\mathrm{C} 12-\mathrm{C} 11-\mathrm{N} 3$ & $122.9(2)$ & $\mathrm{H} 19 \mathrm{~B}-\mathrm{C} 19-\mathrm{H} 19 \mathrm{C}$ & 109.5 \\
\hline $\mathrm{C} 12-\mathrm{C} 11-\mathrm{C} 10$ & $116.1(3)$ & $\mathrm{N} 2-\mathrm{N} 1-\mathrm{S} 1$ & $114.78(19)$ \\
\hline $\mathrm{N} 3-\mathrm{C} 11-\mathrm{C} 10$ & $121.0(3)$ & $\mathrm{N} 2-\mathrm{N} 1-\mathrm{H} 1 \mathrm{~N}$ & $117(2)$ \\
\hline $\mathrm{C} 13-\mathrm{C} 12-\mathrm{C} 11$ & $121.7(2)$ & $\mathrm{S} 1-\mathrm{N} 1-\mathrm{H} 1 \mathrm{~N}$ & $115(2)$ \\
\hline $\mathrm{C} 13-\mathrm{C} 12-\mathrm{H} 12$ & 119.2 & $\mathrm{C} 7-\mathrm{N} 2-\mathrm{N} 1$ & $116.0(2)$ \\
\hline $\mathrm{C} 11-\mathrm{C} 12-\mathrm{H} 12$ & 119.2 & $\mathrm{C} 11-\mathrm{N} 3-\mathrm{C} 18$ & $116.7(2)$ \\
\hline $\mathrm{C} 8-\mathrm{C} 13-\mathrm{C} 12$ & $121.9(3)$ & $\mathrm{C} 11-\mathrm{N} 3-\mathrm{C} 14$ & $116.3(2)$ \\
\hline $\mathrm{C} 8-\mathrm{C} 13-\mathrm{H} 13$ & 119.0 & $\mathrm{C} 18-\mathrm{N} 3-\mathrm{C} 14$ & $114.8(2)$ \\
\hline $\mathrm{C} 12-\mathrm{C} 13-\mathrm{H} 13$ & 119.0 & $\mathrm{O} 1-\mathrm{S} 1-\mathrm{O} 2$ & $120.42(13)$ \\
\hline $\mathrm{N} 3-\mathrm{C} 14-\mathrm{C} 15$ & $114.6(3)$ & $\mathrm{O} 1-\mathrm{S} 1-\mathrm{N} 1$ & $104.23(13)$ \\
\hline $\mathrm{N} 3-\mathrm{C} 14-\mathrm{H} 14 \mathrm{~A}$ & 108.6 & $\mathrm{O} 2-\mathrm{S} 1-\mathrm{N} 1$ & $107.07(13)$ \\
\hline $\mathrm{C} 15-\mathrm{C} 14-\mathrm{H} 14 \mathrm{~A}$ & 108.6 & $\mathrm{O} 1-\mathrm{S} 1-\mathrm{C} 1$ & $108.61(12)$ \\
\hline N3-C14-H14B & 108.6 & $\mathrm{O} 2-\mathrm{S} 1-\mathrm{C} 1$ & $107.88(13)$ \\
\hline $\mathrm{C} 15-\mathrm{C} 14-\mathrm{H} 14 \mathrm{~B}$ & 108.6 & $\mathrm{~N} 1-\mathrm{S} 1-\mathrm{C} 1$ & $108.05(12)$ \\
\hline $\mathrm{C} 6-\mathrm{C} 1-\mathrm{C} 2-\mathrm{C} 3$ & $1.3(4)$ & $\mathrm{C} 14-\mathrm{C} 15-\mathrm{C} 16-\mathrm{C} 17$ & $-51.1(5)$ \\
\hline $\mathrm{S} 1-\mathrm{C} 1-\mathrm{C} 2-\mathrm{C} 3$ & $-177.0(2)$ & $\mathrm{C} 15-\mathrm{C} 16-\mathrm{C} 17-\mathrm{C} 18$ & $51.3(5)$ \\
\hline $\mathrm{C} 1-\mathrm{C} 2-\mathrm{C} 3-\mathrm{C} 4$ & $-0.3(5)$ & $\mathrm{C} 16-\mathrm{C} 17-\mathrm{C} 18-\mathrm{N} 3$ & $-48.4(5)$ \\
\hline $\mathrm{C} 2-\mathrm{C} 3-\mathrm{C} 4-\mathrm{C} 5$ & $-0.7(4)$ & $\mathrm{C} 8-\mathrm{C} 7-\mathrm{N} 2-\mathrm{N} 1$ & $-176.9(2)$ \\
\hline $\mathrm{C} 2-\mathrm{C} 3-\mathrm{C} 4-\mathrm{C} 19$ & $178.2(3)$ & $\mathrm{S} 1-\mathrm{N} 1-\mathrm{N} 2-\mathrm{C} 7$ & $-168.48(19)$ \\
\hline $\mathrm{C} 3-\mathrm{C} 4-\mathrm{C} 5-\mathrm{C} 6$ & $0.7(4)$ & $\mathrm{C} 12-\mathrm{C} 11-\mathrm{N} 3-\mathrm{C} 18$ & $-1.2(4)$ \\
\hline $\mathrm{C} 19-\mathrm{C} 4-\mathrm{C} 5-\mathrm{C} 6$ & $-178.2(3)$ & $\mathrm{C} 10-\mathrm{C} 11-\mathrm{N} 3-\mathrm{C} 18$ & $177.5(3)$ \\
\hline $\mathrm{C} 4-\mathrm{C} 5-\mathrm{C} 6-\mathrm{C} 1$ & $0.3(4)$ & $\mathrm{C} 12-\mathrm{C} 11-\mathrm{N} 3-\mathrm{C} 14$ & $139.5(3)$ \\
\hline $\mathrm{C} 2-\mathrm{C} 1-\mathrm{C} 6-\mathrm{C} 5$ & $-1.3(4)$ & $\mathrm{C} 10-\mathrm{C} 11-\mathrm{N} 3-\mathrm{C} 14$ & $-41.8(4)$ \\
\hline $\mathrm{S} 1-\mathrm{C} 1-\mathrm{C} 6-\mathrm{C} 5$ & $177.1(2)$ & $\mathrm{C} 17-\mathrm{C} 18-\mathrm{N} 3-\mathrm{C} 11$ & $-174.8(3)$ \\
\hline $\mathrm{N} 2-\mathrm{C} 7-\mathrm{C} 8-\mathrm{C} 13$ & $171.5(3)$ & $\mathrm{C} 17-\mathrm{C} 18-\mathrm{N} 3-\mathrm{C} 14$ & $43.9(4)$ \\
\hline $\mathrm{N} 2-\mathrm{C} 7-\mathrm{C} 8-\mathrm{C} 9$ & $-6.2(4)$ & $\mathrm{C} 15-\mathrm{C} 14-\mathrm{N} 3-\mathrm{C} 11$ & $175.2(3)$ \\
\hline $\mathrm{C} 13-\mathrm{C} 8-\mathrm{C} 9-\mathrm{C} 10$ & $0.6(4)$ & $\mathrm{C} 15-\mathrm{C} 14-\mathrm{N} 3-\mathrm{C} 18$ & $-43.4(4)$ \\
\hline $\mathrm{C} 7-\mathrm{C} 8-\mathrm{C} 9-\mathrm{C} 10$ & $178.4(3)$ & $\mathrm{N} 2-\mathrm{N} 1-\mathrm{S} 1-\mathrm{O} 1$ & $178.95(18)$ \\
\hline $\mathrm{C} 8-\mathrm{C} 9-\mathrm{C} 10-\mathrm{C} 11$ & $-0.4(4)$ & $\mathrm{N} 2-\mathrm{N} 1-\mathrm{S} 1-\mathrm{O} 2$ & $-52.4(2)$ \\
\hline
\end{tabular}




$\begin{array}{llll}\mathrm{C} 9-\mathrm{C} 10-\mathrm{C} 11-\mathrm{C} 12 & -0.1(4) & \mathrm{N} 2-\mathrm{N} 1-\mathrm{S} 1-\mathrm{C} 1 & 63.5(2) \\ \mathrm{C} 9-\mathrm{C} 10-\mathrm{C} 11-\mathrm{N} 3 & -178.9(3) & \mathrm{C} 2-\mathrm{C} 1-\mathrm{S} 1-\mathrm{O} 1 & 146.9(2) \\ \mathrm{N} 3-\mathrm{C} 11-\mathrm{C} 12-\mathrm{C} 13 & 179.0(3) & \mathrm{C} 6-\mathrm{C} 1-\mathrm{S} 1-\mathrm{O} 1 & -31.4(2) \\ \mathrm{C} 10-\mathrm{C} 11-\mathrm{C} 12-\mathrm{C} 13 & 0.2(4) & \mathrm{C} 2-\mathrm{C} 1-\mathrm{S} 1-\mathrm{O} 2 & 14.9(2) \\ \mathrm{C} 9-\mathrm{C} 8-\mathrm{C} 13-\mathrm{C} 12 & -0.5(4) & \mathrm{C} 6-\mathrm{C} 1-\mathrm{S} 1-\mathrm{O} 2 & -163.5(2) \\ \mathrm{C} 7-\mathrm{C} 8-\mathrm{C} 13-\mathrm{C} 12 & -178.3(3) & \mathrm{C} 2-\mathrm{C} 1-\mathrm{S} 1-\mathrm{N} 1 & -100.6(2) \\ \mathrm{C} 11-\mathrm{C} 12-\mathrm{C} 13-\mathrm{C} 8 & 0.1(4) & \mathrm{C} 6-\mathrm{C} 1-\mathrm{S} 1-\mathrm{N} 1 & 81.1(2) \\ \mathrm{N} 3-\mathrm{C} 14-\mathrm{C} 15-\mathrm{C} 16 & 47.7(5) & & \end{array}$

Hydrogen-bond geometry $\left(\AA,{ }^{\circ}\right)$

\begin{tabular}{lllll}
\hline$D-\mathrm{H} \cdots A$ & $D-\mathrm{H}$ & $\mathrm{H} \cdots A$ & $D \cdots A$ & $D-\mathrm{H} \cdots A$ \\
\hline $\mathrm{N} 1-\mathrm{H} 1 N \cdots \mathrm{O} 2^{\mathrm{i}}$ & $0.79(3)$ & $2.29(3)$ & $3.068(3)$ & $170(3)$ \\
\hline
\end{tabular}

Symmetry code: (i) $x, y+1, z$.

(E)-4-Chloro-N'-[4-(piperidin-1-yl)benzylidene] benzenesulfonohydrazide (III)

Crystal data

$\mathrm{C}_{18} \mathrm{H}_{20} \mathrm{ClN}_{3} \mathrm{O}_{2} \mathrm{~S}$

$M_{r}=377.88$

Monoclinic, $C 2 / c$

$a=33.052(6) \AA$

$b=5.258(1) \AA$

$c=43.026(8) \AA$

$\beta=94.05(2)^{\circ}$

$V=7459(2) \AA^{3}$

$Z=16$

\section{Data collection}

Oxford Diffraction Xcalibur diffractometer with Sapphire CCD

Radiation source: Enhance (Mo) X-ray Source

Rotation method data acquisition using $\omega$ scans.

Absorption correction: multi-scan

(CrysAlis RED; Oxford Diffraction, 2009)

$T_{\min }=0.851, T_{\max }=0.955$

14002 measured reflections

\section{Refinement}

Refinement on $F^{2}$

Least-squares matrix: full

$R\left[F^{2}>2 \sigma\left(F^{2}\right)\right]=0.075$

$w R\left(F^{2}\right)=0.172$

$S=1.01$

6833 reflections

457 parameters

31 restraints
$F(000)=3168$

$D_{\mathrm{x}}=1.346 \mathrm{Mg} \mathrm{m}^{-3}$

Mo $K \alpha$ radiation, $\lambda=0.71073 \AA$

Cell parameters from 1174 reflections

$\theta=2.5-27.8^{\circ}$

$\mu=0.33 \mathrm{~mm}^{-1}$

$T=293 \mathrm{~K}$

Prism, red

$0.50 \times 0.26 \times 0.14 \mathrm{~mm}$

6833 independent reflections

2999 reflections with $I>2 \sigma(I)$

$R_{\text {int }}=0.042$

$\theta_{\max }=25.4^{\circ}, \theta_{\min }=2.7^{\circ}$

$h=-39 \rightarrow 39$

$k=-6 \rightarrow 6$

$l=-42 \rightarrow 51$

Hydrogen site location: mixed

$\mathrm{H}$ atoms treated by a mixture of independent and constrained refinement

$w=1 /\left[\sigma^{2}\left(F_{\mathrm{o}}^{2}\right)+(0.0483 P)^{2}+15.4136 P\right]$

where $P=\left(F_{\mathrm{o}}{ }^{2}+2 F_{\mathrm{c}}{ }^{2}\right) / 3$

$(\Delta / \sigma)_{\max }<0.001$

$\Delta \rho_{\max }=0.27 \mathrm{e} \AA^{-3}$

$\Delta \rho_{\min }=-0.30$ e $\AA^{-3}$ 


\section{Special details}

Geometry. All esds (except the esd in the dihedral angle between two 1.s. planes) are estimated using the full covariance matrix. The cell esds are taken into account individually in the estimation of esds in distances, angles and torsion angles; correlations between esds in cell parameters are only used when they are defined by crystal symmetry. An approximate (isotropic) treatment of cell esds is used for estimating esds involving l.s. planes.

Fractional atomic coordinates and isotropic or equivalent isotropic displacement parameters $\left(\AA^{2}\right)$

\begin{tabular}{|c|c|c|c|c|}
\hline & $x$ & $y$ & $z$ & $U_{\text {iso }} * / U_{\text {eq }}$ \\
\hline $\mathrm{Cl1}$ & $0.47550(5)$ & $0.3259(4)$ & $0.18187(4)$ & $0.1273(7)$ \\
\hline S1 & $0.31044(4)$ & $0.0442(3)$ & $0.11230(3)$ & 0.0657 (4) \\
\hline $\mathrm{O} 1$ & $0.27796(10)$ & $0.1162(6)$ & $0.13046(7)$ & $0.0779(10)$ \\
\hline $\mathrm{O} 2$ & $0.31308(11)$ & $-0.2078(6)$ & $0.10041(8)$ & $0.0797(10)$ \\
\hline N1 & 0.30660 (14) & $0.2366(8)$ & $0.08245(9)$ & $0.0636(12)$ \\
\hline $\mathrm{H} 1 \mathrm{~N}$ & $0.2999(15)$ & $0.387(9)$ & $0.0854(11)$ & $0.076^{*}$ \\
\hline $\mathrm{N} 2$ & $0.33782(12)$ & $0.2115(8)$ & $0.06209(9)$ & $0.0625(11)$ \\
\hline N3 & $0.45666(13)$ & $0.3870(8)$ & $-0.04820(10)$ & $0.0770(12)$ \\
\hline $\mathrm{C} 1$ & $0.35682(15)$ & $0.1126(9)$ & $0.13273(10)$ & $0.0563(13)$ \\
\hline $\mathrm{C} 2$ & $0.39197(19)$ & $-0.0048(11)$ & $0.12540(13)$ & $0.0843(17)$ \\
\hline $\mathrm{H} 2$ & 0.3912 & -0.1276 & 0.1098 & $0.101 *$ \\
\hline $\mathrm{C} 3$ & $0.42848(19)$ & $0.0587(12)$ & $0.14107(15)$ & 0.0940 (19) \\
\hline $\mathrm{H} 3$ & 0.4523 & -0.0232 & 0.1365 & $0.113^{*}$ \\
\hline $\mathrm{C} 4$ & $0.42913(17)$ & $0.2419(12)$ & $0.16323(12)$ & $0.0763(16)$ \\
\hline $\mathrm{C} 5$ & $0.3948(2)$ & $0.3541(12)$ & $0.17136(13)$ & $0.0895(18)$ \\
\hline H5 & 0.3958 & 0.4727 & 0.1874 & $0.107 *$ \\
\hline C6 & $0.35822(17)$ & $0.2929(11)$ & $0.15586(12)$ & $0.0813(16)$ \\
\hline H6 & 0.3346 & 0.3735 & 0.1610 & $0.098^{*}$ \\
\hline $\mathrm{C} 7$ & $0.34159(15)$ & $0.3963(10)$ & $0.04343(11)$ & $0.0619(13)$ \\
\hline $\mathrm{H} 7$ & 0.3245 & 0.5362 & 0.0445 & $0.074 *$ \\
\hline $\mathrm{C} 8$ & $0.37184(15)$ & $0.3948(9)$ & $0.02046(10)$ & $0.0565(13)$ \\
\hline C9 & $0.40063(17)$ & $0.2080(10)$ & $0.01923(12)$ & $0.0733(15)$ \\
\hline H9 & 0.4013 & 0.0785 & 0.0340 & $0.088^{*}$ \\
\hline $\mathrm{C} 10$ & $0.42840(16)$ & $0.2054(10)$ & $-0.00296(12)$ & $0.0726(15)$ \\
\hline H10 & 0.4473 & 0.0747 & -0.0031 & $0.087^{*}$ \\
\hline C11 & $0.42853(15)$ & $0.3964(10)$ & $-0.02534(11)$ & $0.0589(13)$ \\
\hline $\mathrm{C} 12$ & $0.39956(16)$ & $0.5837(10)$ & $-0.02398(11)$ & $0.0686(14)$ \\
\hline H12 & 0.3986 & 0.7135 & -0.0387 & $0.082 *$ \\
\hline $\mathrm{C} 13$ & $0.37211(16)$ & $0.5843(9)$ & $-0.00157(11)$ & $0.0689(14)$ \\
\hline H13 & 0.3533 & 0.7155 & -0.0012 & $0.083^{*}$ \\
\hline C14 & 0.49585 (19) & $0.3073(16)$ & $-0.04105(16)$ & $0.143(3)$ \\
\hline H14A & 0.4943 & 0.1452 & -0.0304 & $0.172 *$ \\
\hline H14B & 0.5081 & 0.4269 & -0.0260 & $0.172 *$ \\
\hline $\mathrm{C} 15$ & $0.5239(2)$ & $0.2760(15)$ & $-0.06531(18)$ & $0.139(3)$ \\
\hline H15A & 0.5512 & 0.3004 & -0.0560 & $0.166^{*}$ \\
\hline H15B & 0.5220 & 0.1017 & -0.0727 & $0.166^{*}$ \\
\hline $\mathrm{C} 16$ & $0.5182(2)$ & $0.4427(14)$ & $-0.09203(15)$ & $0.118(2)$ \\
\hline H16A & 0.5346 & 0.5939 & -0.0881 & $0.142 *$ \\
\hline H16B & 0.5282 & 0.3573 & -0.1100 & $0.142^{*}$ \\
\hline
\end{tabular}




\begin{tabular}{|c|c|c|c|c|}
\hline C17 & $0.4780(2)$ & $0.5174(17)$ & $-0.09945(16)$ & $0.150(3)$ \\
\hline H17A & 0.4658 & 0.3929 & -0.1139 & $0.180^{*}$ \\
\hline H17B & 0.4787 & 0.6774 & -0.1106 & $0.180^{*}$ \\
\hline $\mathrm{C} 18$ & 0.45054 (18) & $0.5500(13)$ & $-0.07439(13)$ & $0.115(2)$ \\
\hline H18A & 0.4528 & 0.7243 & -0.0671 & $0.138^{*}$ \\
\hline H18B & 0.4230 & 0.5263 & -0.0832 & $0.138^{*}$ \\
\hline $\mathrm{Cl} 2$ & $0.22664(5)$ & $0.4803(4)$ & $0.02015(4)$ & $0.1245(7)$ \\
\hline S2 & $0.13541(5)$ & $0.7140(3)$ & $0.13800(3)$ & $0.0696(4)$ \\
\hline $\mathrm{O} 3$ & $0.09541(11)$ & $0.6126(7)$ & $0.13364(8)$ & $0.0883(11)$ \\
\hline $\mathrm{O} 4$ & $0.14190(11)$ & $0.9734(6)$ & $0.14659(7)$ & $0.0845(11)$ \\
\hline N4 & $0.15747(15)$ & $0.5383(8)$ & $0.16570(10)$ & $0.0702(13)$ \\
\hline $\mathrm{H} 4 \mathrm{~N}$ & $0.1518(16)$ & $0.384(9)$ & $0.1629(12)$ & $0.084 *$ \\
\hline N5 & $0.19715(14)$ & $0.6102(8)$ & $0.17526(9)$ & $0.0654(11)$ \\
\hline N6 & $0.37521(14)$ & $0.6426(8)$ & $0.24681(10)$ & $0.0726(12)$ \\
\hline C19 & $0.16088(15)$ & $0.6564(9)$ & $0.10444(10)$ & $0.0576(13)$ \\
\hline $\mathrm{C} 20$ & $0.19395(16)$ & $0.7992(11)$ & $0.09801(12)$ & $0.0746(15)$ \\
\hline $\mathrm{H} 20$ & 0.2028 & 0.9306 & 0.1113 & $0.090^{*}$ \\
\hline $\mathrm{C} 21$ & $0.21407(16)$ & $0.7463(12)$ & $0.07161(13)$ & $0.0823(17)$ \\
\hline $\mathrm{H} 21$ & 0.2361 & 0.8446 & 0.0666 & $0.099 *$ \\
\hline $\mathrm{C} 22$ & $0.20116(18)$ & $0.5481(12)$ & $0.05306(11)$ & $0.0748(16)$ \\
\hline $\mathrm{C} 23$ & $0.16849(19)$ & $0.4049(11)$ & $0.05942(13)$ & $0.0823(17)$ \\
\hline $\mathrm{H} 23$ & 0.1600 & 0.2722 & 0.0462 & $0.099 *$ \\
\hline $\mathrm{C} 24$ & $0.14812(17)$ & $0.4565(10)$ & $0.08532(12)$ & $0.0771(16)$ \\
\hline H24 & 0.1259 & 0.3583 & 0.0900 & $0.093 *$ \\
\hline $\mathrm{C} 25$ & $0.21791(15)$ & $0.4430(10)$ & $0.19052(10)$ & $0.0606(13)$ \\
\hline $\mathrm{H} 25$ & 0.2068 & 0.2826 & 0.1932 & $0.073 *$ \\
\hline $\mathrm{C} 26$ & $0.25843(15)$ & $0.4968(9)$ & $0.20382(10)$ & $0.0551(12)$ \\
\hline $\mathrm{C} 27$ & $0.28111(17)$ & $0.7029(10)$ & $0.19542(10)$ & $0.0654(14)$ \\
\hline $\mathrm{H} 27$ & 0.2704 & 0.8106 & 0.1798 & $0.078 *$ \\
\hline $\mathrm{C} 28$ & $0.31893(17)$ & $0.7547(10)$ & $0.20933(11)$ & $0.0692(14)$ \\
\hline $\mathrm{H} 28$ & 0.3333 & 0.8946 & 0.2028 & $0.083 *$ \\
\hline $\mathrm{C} 29$ & $0.33619(16)$ & $0.5996(10)$ & $0.23328(11)$ & 0.0609 (13) \\
\hline C30 & $0.31319(16)$ & $0.3937(10)$ & $0.24176(11)$ & $0.0675(14)$ \\
\hline $\mathrm{H} 30$ & 0.3235 & 0.2879 & 0.2577 & $0.081 *$ \\
\hline $\mathrm{C} 31$ & 0.27607 (16) & $0.3414(10)$ & $0.22745(11)$ & $0.0665(14)$ \\
\hline H31 & 0.2621 & 0.1985 & 0.2335 & $0.080^{*}$ \\
\hline C32 & 0.39719 (19) & $0.8645(12)$ & $0.23767(15)$ & $0.104(2)$ \\
\hline $\mathrm{H} 32 \mathrm{~A}$ & 0.3844 & 1.0147 & 0.2457 & $0.125^{*}$ \\
\hline H32B & 0.3951 & 0.8759 & 0.2151 & $0.125^{*}$ \\
\hline C33 & $0.4411(2)$ & $0.8653(14)$ & $0.24890(18)$ & $0.123(2)$ \\
\hline $\mathrm{H} 33 \mathrm{~A}$ & 0.4553 & 0.7399 & 0.2372 & $0.148^{*}$ \\
\hline H33B & 0.4525 & 1.0307 & 0.2447 & $0.148^{*}$ \\
\hline C34 & 0.44807 (19) & 0.8091 (14) & $0.28267(17)$ & $0.110(2)$ \\
\hline $\mathrm{H} 34 \mathrm{~A}$ & 0.4769 & 0.7871 & 0.2879 & $0.132 *$ \\
\hline H34B & 0.4388 & 0.9514 & 0.2946 & $0.132 *$ \\
\hline C35 & 0.42629 (19) & 0.5767 (14) & $0.29094(15)$ & $0.117(2)$ \\
\hline $\mathrm{H} 35 \mathrm{~A}$ & 0.4279 & 0.5591 & 0.3134 & $0.140 *$ \\
\hline H35B & 0.4395 & 0.4302 & 0.2824 & $0.140^{*}$ \\
\hline
\end{tabular}




$\begin{array}{lllll}\text { C36 } & 0.38288(17) & 0.5786(13) & 0.27912(12) & 0.099(2) \\ \text { H36A } & 0.3716 & 0.4115 & 0.2825 & 0.119^{*} \\ \text { H36B } & 0.3685 & 0.6990 & 0.2914 & 0.119^{*}\end{array}$

Atomic displacement parameters $\left(\AA^{2}\right)$

\begin{tabular}{|c|c|c|c|c|c|c|}
\hline & $U^{11}$ & $U^{22}$ & $U^{33}$ & $U^{12}$ & $U^{13}$ & $U^{23}$ \\
\hline $\mathrm{Cl1}$ & $0.0949(12)$ & $0.1716(18)$ & $0.1116(13)$ & $-0.0191(13)$ & $-0.0185(10)$ & $-0.0055(13)$ \\
\hline S1 & $0.0844(10)$ & $0.0535(9)$ & $0.0593(8)$ & $-0.0097(8)$ & $0.0064(7)$ & $0.0000(7)$ \\
\hline $\mathrm{O} 1$ & 0.077 (2) & $0.083(3)$ & $0.076(2)$ & $-0.012(2)$ & $0.022(2)$ & $0.000(2)$ \\
\hline $\mathrm{O} 2$ & $0.108(3)$ & $0.045(2)$ & $0.086(2)$ & $-0.013(2)$ & $0.002(2)$ & $-0.0052(18)$ \\
\hline N1 & $0.083(3)$ & $0.054(3)$ & $0.054(2)$ & $0.005(3)$ & $0.003(2)$ & $0.007(2)$ \\
\hline $\mathrm{N} 2$ & $0.080(3)$ & $0.058(3)$ & $0.050(2)$ & $-0.004(2)$ & $0.004(2)$ & $-0.002(2)$ \\
\hline N3 & 0.077 (2) & 0.077 (3) & $0.077(3)$ & $0.010(2)$ & $0.003(2)$ & $0.017(2)$ \\
\hline $\mathrm{C} 1$ & $0.076(4)$ & $0.048(3)$ & $0.046(3)$ & $-0.007(3)$ & $0.014(2)$ & $-0.001(2)$ \\
\hline $\mathrm{C} 2$ & $0.093(4)$ & $0.080(4)$ & $0.081(4)$ & $0.011(4)$ & $0.010(4)$ & $-0.025(3)$ \\
\hline $\mathrm{C} 3$ & $0.080(4)$ & $0.105(5)$ & $0.098(5)$ & $0.019(4)$ & $0.010(4)$ & $-0.016(4)$ \\
\hline $\mathrm{C} 4$ & $0.077(4)$ & $0.089(5)$ & $0.062(3)$ & -0.009 & $0.004(3)$ & $0.000(3)$ \\
\hline $\mathrm{C} 5$ & $0.092(5)$ & $0.101(5)$ & $0.075(4)$ & $-0.002(4)$ & $0.001(4)$ & $-0.031(3)$ \\
\hline C6 & $0.083(4)$ & $0.090(4)$ & $0.071(4)$ & $0.006(4)$ & $0.009(3)$ & $-0.023(3)$ \\
\hline $\mathrm{C} 7$ & $0.073(4)$ & $0.058(3)$ & $0.053(3)$ & $-0.001(3)$ & $-0.004(3)$ & $0.001(3)$ \\
\hline $\mathrm{C} 8$ & $0.070(3)$ & $0.050(3)$ & $0.048(3)$ & $-0.005(3)$ & $-0.006(3)$ & $0.001(3)$ \\
\hline $\mathrm{C} 9$ & $0.093(4)$ & $0.062(4)$ & $0.064(3)$ & $0.011(4)$ & $0.001(3)$ & $0.021(3)$ \\
\hline $\mathrm{C} 10$ & $0.081(4)$ & $0.063(4)$ & $0.074(4)$ & $0.021(3)$ & $0.008(3)$ & $0.015(3)$ \\
\hline C11 & $0.062(3)$ & 0.059 (3) & $0.056(3)$ & $0.000(3)$ & $0.000(3)$ & $0.002(3)$ \\
\hline C12 & $0.079(4)$ & $0.065(4)$ & $0.062(3)$ & $0.014(3)$ & $0.005(3)$ & $0.019(3)$ \\
\hline $\mathrm{C} 13$ & $0.080(4)$ & $0.059(3)$ & $0.068(3)$ & $0.017(3)$ & $0.011(3)$ & $0.013(3)$ \\
\hline C14 & $0.100(3)$ & $0.204(7)$ & $0.128(5)$ & $0.073(5)$ & $0.031(4)$ & $0.061(5)$ \\
\hline $\mathrm{C} 15$ & $0.114(5)$ & $0.156(6)$ & $0.152(6)$ & $0.052(5)$ & $0.049(5)$ & $0.043(5)$ \\
\hline $\mathrm{C} 16$ & $0.119(4)$ & $0.127(6)$ & $0.113(5)$ & $0.031(5)$ & $0.047(4)$ & $0.026(5)$ \\
\hline $\mathrm{C} 17$ & $0.122(4)$ & $0.220(7)$ & $0.115(4)$ & $0.048(5)$ & $0.054(3)$ & $0.062(5)$ \\
\hline C18 & $0.108(4)$ & $0.149(5)$ & $0.091(4)$ & $0.041(4)$ & $0.032(3)$ & $0.054(3)$ \\
\hline $\mathrm{Cl} 2$ & 0.1149 (13) & 0.1825 (19) & $0.0782(10)$ & $0.0329(13)$ & $0.0209(9)$ & $-0.0211(12)$ \\
\hline $\mathrm{S} 2$ & $0.0840(11)$ & $0.0686(10)$ & $0.0560(8)$ & $0.0118(9)$ & $0.0033(7)$ & 0.0047 (7) \\
\hline $\mathrm{O} 3$ & $0.069(2)$ & $0.107(3)$ & $0.088(3)$ & $0.003(2)$ & $0.003(2)$ & $0.009(2)$ \\
\hline $\mathrm{O} 4$ & $0.122(3)$ & $0.062(2)$ & $0.070(2)$ & $0.019(2)$ & $0.012(2)$ & $-0.0044(19)$ \\
\hline N4 & $0.083(3)$ & $0.068(3)$ & $0.058(3)$ & $-0.002(3)$ & -0.001 (2) & 0.008 (2) \\
\hline N5 & 0.080 & $0.064(3)$ & $0.051(2)$ & $0.005(3)$ & $-0.002(2)$ & $0.002(2)$ \\
\hline N6 & $0.078(3)$ & $0.074(3)$ & $0.067(3)$ & $-0.002(3)$ & $0.015(2)$ & $0.013(2)$ \\
\hline C19 & 0.068 & $0.054(3)$ & 0.049 & $0.001(3)$ & $-0.006(2)$ & $0.001(3)$ \\
\hline $\mathrm{C} 20$ & $0.081(4)$ & $0.080(4)$ & $0.062(4)$ & -0.008 & -0.004 (3) & -0.014 (3) \\
\hline $\mathrm{C} 21$ & $0.076(4)$ & $0.097(5)$ & $0.074(4)$ & $-0.011(4)$ & $0.004(3)$ & $0.006(4)$ \\
\hline $\mathrm{C} 22$ & $0.080(4)$ & $0.090(4)$ & $0.053(3)$ & $0.020(4)$ & $-0.002(3)$ & $-0.006(3)$ \\
\hline $\mathrm{C} 23$ & $0.105(5)$ & $0.070(4)$ & $0.070(4)$ & $0.000(4)$ & $-0.005(4)$ & $-0.012(3)$ \\
\hline $\mathrm{C} 24$ & $0.104(4)$ & $0.065(4)$ & $0.063(3)$ & -0.019 & $0.005(3)$ & $0.001(3)$ \\
\hline $\mathrm{C} 25$ & $0.072(4)$ & $0.056(3)$ & $0.056(3)$ & $0.002(3)$ & $0.016(3)$ & 0.000 \\
\hline $\mathrm{C} 26$ & $0.072(4)$ & $0.047(3)$ & $0.048(3)$ & 0.005 & $0.017(3)$ & $0.000(2)$ \\
\hline $\mathrm{C} 27$ & $0.085(4)$ & $0.064(4)$ & $0.047(3)$ & $0.006(3)$ & 0.007 (3) & $0.005(3)$ \\
\hline
\end{tabular}




\begin{tabular}{lllllll}
\hline C28 & $0.090(4)$ & $0.058(3)$ & $0.062(3)$ & $-0.007(3)$ & $0.021(3)$ & $0.013(3)$ \\
C29 & $0.070(4)$ & $0.064(4)$ & $0.049(3)$ & $0.008(3)$ & $0.015(3)$ & $-0.005(3)$ \\
C30 & $0.071(4)$ & $0.069(4)$ & $0.064(3)$ & $0.007(3)$ & $0.009(3)$ & $0.021(3)$ \\
C31 & $0.069(4)$ & $0.060(3)$ & $0.073(3)$ & $0.006(3)$ & $0.017(3)$ & $0.015(3)$ \\
C32 & $0.097(5)$ & $0.100(5)$ & $0.114(5)$ & $-0.016(4)$ & $-0.001(4)$ & $0.014(4)$ \\
C33 & $0.090(5)$ & $0.136(6)$ & $0.143(7)$ & $-0.024(5)$ & $0.007(5)$ & $0.022(5)$ \\
C34 & $0.092(5)$ & $0.110(6)$ & $0.127(6)$ & $-0.009(5)$ & $-0.009(4)$ & $-0.012(5)$ \\
C35 & $0.103(5)$ & $0.138(6)$ & $0.107(5)$ & $-0.017(5)$ & $-0.015(4)$ & $0.029(5)$ \\
C36 & $0.086(4)$ & $0.143(6)$ & $0.068(4)$ & $-0.025(4)$ & $0.000(3)$ & $0.006(4)$ \\
\hline
\end{tabular}

Geometric parameters $\left(\AA,{ }^{\circ}\right)$

\begin{tabular}{|c|c|c|c|}
\hline $\mathrm{Cl1}-\mathrm{C} 4$ & $1.736(5)$ & $\mathrm{C} 12-\mathrm{C} 22$ & $1.735(5)$ \\
\hline $\mathrm{S} 1-\mathrm{O} 1$ & $1.423(3)$ & $\mathrm{S} 2-\mathrm{O} 4$ & $1.425(3)$ \\
\hline $\mathrm{S} 1-\mathrm{O} 2$ & $1.425(3)$ & $\mathrm{S} 2-\mathrm{O} 3$ & $1.426(4)$ \\
\hline $\mathrm{S} 1-\mathrm{N} 1$ & $1.633(4)$ & $\mathrm{S} 2-\mathrm{N} 4$ & $1.638(4)$ \\
\hline $\mathrm{S} 1-\mathrm{C} 1$ & $1.750(5)$ & $\mathrm{S} 2-\mathrm{C} 19$ & $1.748(5)$ \\
\hline $\mathrm{N} 1-\mathrm{N} 2$ & $1.406(5)$ & $\mathrm{N} 4-\mathrm{N} 5$ & $1.399(5)$ \\
\hline $\mathrm{N} 1-\mathrm{H} 1 \mathrm{~N}$ & $0.83(4)$ & $\mathrm{N} 4-\mathrm{H} 4 \mathrm{~N}$ & $0.84(5)$ \\
\hline $\mathrm{N} 2-\mathrm{C} 7$ & $1.272(5)$ & N5-C25 & $1.269(5)$ \\
\hline $\mathrm{N} 3-\mathrm{C} 14$ & $1.375(6)$ & $\mathrm{N} 6-\mathrm{C} 29$ & $1.395(6)$ \\
\hline $\mathrm{N} 3-\mathrm{C} 11$ & $1.401(6)$ & $\mathrm{N} 6-\mathrm{C} 36$ & $1.435(6)$ \\
\hline $\mathrm{N} 3-\mathrm{C} 18$ & $1.419(6)$ & $\mathrm{N} 6-\mathrm{C} 32$ & $1.444(6)$ \\
\hline $\mathrm{C} 1-\mathrm{C} 2$ & $1.371(6)$ & $\mathrm{C} 19-\mathrm{C} 20$ & $1.371(6)$ \\
\hline $\mathrm{C} 1-\mathrm{C} 6$ & $1.373(6)$ & $\mathrm{C} 19-\mathrm{C} 24$ & $1.382(6)$ \\
\hline $\mathrm{C} 2-\mathrm{C} 3$ & $1.381(7)$ & $\mathrm{C} 20-\mathrm{C} 21$ & $1.384(7)$ \\
\hline $\mathrm{C} 2-\mathrm{H} 2$ & 0.9300 & $\mathrm{C} 20-\mathrm{H} 20$ & 0.9300 \\
\hline $\mathrm{C} 3-\mathrm{C} 4$ & $1.355(7)$ & $\mathrm{C} 21-\mathrm{C} 22$ & $1.363(7)$ \\
\hline $\mathrm{C} 3-\mathrm{H} 3$ & 0.9300 & $\mathrm{C} 21-\mathrm{H} 21$ & 0.9300 \\
\hline $\mathrm{C} 4-\mathrm{C} 5$ & $1.347(7)$ & $\mathrm{C} 22-\mathrm{C} 23$ & $1.360(7)$ \\
\hline $\mathrm{C} 5-\mathrm{C} 6$ & $1.377(7)$ & $\mathrm{C} 23-\mathrm{C} 24$ & $1.369(7)$ \\
\hline C5-H5 & 0.9300 & $\mathrm{C} 23-\mathrm{H} 23$ & 0.9300 \\
\hline C6-H6 & 0.9300 & $\mathrm{C} 24-\mathrm{H} 24$ & 0.9300 \\
\hline $\mathrm{C} 7-\mathrm{C} 8$ & $1.455(6)$ & $\mathrm{C} 25-\mathrm{C} 26$ & $1.447(6)$ \\
\hline $\mathrm{C} 7-\mathrm{H} 7$ & 0.9300 & $\mathrm{C} 25-\mathrm{H} 25$ & 0.9300 \\
\hline $\mathrm{C} 8-\mathrm{C} 9$ & $1.371(6)$ & $\mathrm{C} 26-\mathrm{C} 27$ & $1.380(6)$ \\
\hline $\mathrm{C} 8-\mathrm{C} 13$ & $1.376(6)$ & $\mathrm{C} 26-\mathrm{C} 31$ & $1.399(6)$ \\
\hline $\mathrm{C} 9-\mathrm{C} 10$ & $1.370(6)$ & $\mathrm{C} 27-\mathrm{C} 28$ & $1.374(6)$ \\
\hline C9-H9 & 0.9300 & $\mathrm{C} 27-\mathrm{H} 27$ & 0.9300 \\
\hline $\mathrm{C} 10-\mathrm{C} 11$ & $1.392(6)$ & $\mathrm{C} 28-\mathrm{C} 29$ & $1.403(6)$ \\
\hline $\mathrm{C} 10-\mathrm{H} 10$ & 0.9300 & $\mathrm{C} 28-\mathrm{H} 28$ & 0.9300 \\
\hline $\mathrm{C} 11-\mathrm{C} 12$ & $1.378(6)$ & $\mathrm{C} 29-\mathrm{C} 30$ & $1.386(6)$ \\
\hline $\mathrm{C} 12-\mathrm{C} 13$ & $1.370(6)$ & $\mathrm{C} 30-\mathrm{C} 31$ & $1.361(6)$ \\
\hline $\mathrm{C} 12-\mathrm{H} 12$ & 0.9300 & $\mathrm{C} 30-\mathrm{H} 30$ & 0.9300 \\
\hline $\mathrm{C} 13-\mathrm{H} 13$ & 0.9300 & $\mathrm{C} 31-\mathrm{H} 31$ & 0.9300 \\
\hline $\mathrm{C} 14-\mathrm{C} 15$ & $1.453(7)$ & $\mathrm{C} 32-\mathrm{C} 33$ & $1.497(7)$ \\
\hline $\mathrm{C} 14-\mathrm{H} 14 \mathrm{~A}$ & 0.9700 & $\mathrm{C} 32-\mathrm{H} 32 \mathrm{~A}$ & 0.9700 \\
\hline C14-H14B & 0.9700 & $\mathrm{C} 32-\mathrm{H} 32 \mathrm{~B}$ & 0.9700 \\
\hline
\end{tabular}




\section{$\mathrm{C} 15-\mathrm{C} 16$ \\ C15-H15A \\ C15-H15B \\ $\mathrm{C} 16-\mathrm{C} 17$ \\ C16-H16A \\ C16-H16B \\ $\mathrm{C} 17-\mathrm{C} 18$ \\ C17-H17A \\ C17-H17B \\ C18-H18A \\ C18-H18B}

$\mathrm{O} 1-\mathrm{S} 1-\mathrm{O} 2$

O1-S1-N1

$\mathrm{O} 2-\mathrm{S} 1-\mathrm{N} 1$

$\mathrm{O} 1-\mathrm{S} 1-\mathrm{C} 1$

$\mathrm{O} 2-\mathrm{S} 1-\mathrm{C} 1$

$\mathrm{N} 1-\mathrm{S} 1-\mathrm{C} 1$

$\mathrm{N} 2-\mathrm{N} 1-\mathrm{S} 1$

$\mathrm{N} 2-\mathrm{N} 1-\mathrm{H} 1 \mathrm{~N}$

$\mathrm{S} 1-\mathrm{N} 1-\mathrm{H} 1 \mathrm{~N}$

C7-N2-N1

$\mathrm{C} 14-\mathrm{N} 3-\mathrm{C} 11$

C14-N3-C18

$\mathrm{C} 11-\mathrm{N} 3-\mathrm{C} 18$

$\mathrm{C} 2-\mathrm{C} 1-\mathrm{C} 6$

$\mathrm{C} 2-\mathrm{C} 1-\mathrm{S} 1$

$\mathrm{C} 6-\mathrm{C} 1-\mathrm{S} 1$

$\mathrm{C} 1-\mathrm{C} 2-\mathrm{C} 3$

$\mathrm{C} 1-\mathrm{C} 2-\mathrm{H} 2$

$\mathrm{C} 3-\mathrm{C} 2-\mathrm{H} 2$

$\mathrm{C} 4-\mathrm{C} 3-\mathrm{C} 2$

$\mathrm{C} 4-\mathrm{C} 3-\mathrm{H} 3$

$\mathrm{C} 2-\mathrm{C} 3-\mathrm{H} 3$

$\mathrm{C} 5-\mathrm{C} 4-\mathrm{C} 3$

$\mathrm{C} 5-\mathrm{C} 4-\mathrm{Cl} 1$

$\mathrm{C} 3-\mathrm{C} 4-\mathrm{Cl} 1$

$\mathrm{C} 4-\mathrm{C} 5-\mathrm{C} 6$

$\mathrm{C} 4-\mathrm{C} 5-\mathrm{H} 5$

$\mathrm{C} 6-\mathrm{C} 5-\mathrm{H} 5$

$\mathrm{C} 1-\mathrm{C} 6-\mathrm{C} 5$

$\mathrm{C} 1-\mathrm{C} 6-\mathrm{H} 6$

C5-C6- 66

N2-C7-C8

$\mathrm{N} 2-\mathrm{C} 7-\mathrm{H} 7$

$\mathrm{C} 8-\mathrm{C} 7-\mathrm{H} 7$

$\mathrm{C} 9-\mathrm{C} 8-\mathrm{C} 13$

$\mathrm{C} 9-\mathrm{C} 8-\mathrm{C} 7$
$1.447(8)$

0.9700

0.9700

1.403 (8)

0.9700

0.9700

1.467 (7)

0.9700

0.9700

0.9700

0.9700

$120.8(2)$

104.3 (2)

$107.3(2)$

109.7 (2)

107.3 (2)

106.5 (2)

114.3 (3)

114 (4)

118 (3)

115.5 (4)

$121.0(5)$

$116.2(5)$

118.1 (4)

119.4 (5)

$121.2(4)$

$119.3(4)$

120.3 (5)

119.9

119.9

$119.0(5)$

120.5

120.5

$121.5(5)$

$120.1(5)$

$118.4(5)$

$119.9(5)$

120.0

120.0

$119.7(5)$

120.1

120.1

121.8 (5)

119.1

119.1

$117.0(5)$

$122.8(5)$
$\mathrm{C} 33-\mathrm{C} 34$

C33-H33A

C33-H33B

C $34-C 35$

C34-H34A

C34-H34B

$\mathrm{C} 35-\mathrm{C} 36$

C35-H35A

C35- $\mathrm{H} 35 \mathrm{~B}$

C36- H36A

C36- $336 \mathrm{~B}$

$\mathrm{O} 4-\mathrm{S} 2-\mathrm{O} 3$

$\mathrm{O} 4-\mathrm{S} 2-\mathrm{N} 4$

$\mathrm{O} 3-\mathrm{S} 2-\mathrm{N} 4$

O4-S2-C19

O3-S2-C19

N4-S2-C19

N5-N4-S2

$\mathrm{N} 5-\mathrm{N} 4-\mathrm{H} 4 \mathrm{~N}$

$\mathrm{S} 2-\mathrm{N} 4-\mathrm{H} 4 \mathrm{~N}$

$\mathrm{C} 25-\mathrm{N} 5-\mathrm{N} 4$

$\mathrm{C} 29-\mathrm{N} 6-\mathrm{C} 36$

$\mathrm{C} 29-\mathrm{N} 6-\mathrm{C} 32$

$\mathrm{C} 36-\mathrm{N} 6-\mathrm{C} 32$

$\mathrm{C} 20-\mathrm{C} 19-\mathrm{C} 24$

$\mathrm{C} 20-\mathrm{C} 19-\mathrm{S} 2$

$\mathrm{C} 24-\mathrm{C} 19-\mathrm{S} 2$

$\mathrm{C} 19-\mathrm{C} 20-\mathrm{C} 21$

C19-C20-H20

$\mathrm{C} 21-\mathrm{C} 20-\mathrm{H} 20$

$\mathrm{C} 22-\mathrm{C} 21-\mathrm{C} 20$

$\mathrm{C} 22-\mathrm{C} 21-\mathrm{H} 21$

$\mathrm{C} 20-\mathrm{C} 21-\mathrm{H} 21$

$\mathrm{C} 23-\mathrm{C} 22-\mathrm{C} 21$

$\mathrm{C} 23-\mathrm{C} 22-\mathrm{C} 2$

$\mathrm{C} 21-\mathrm{C} 22-\mathrm{Cl} 2$

$\mathrm{C} 22-\mathrm{C} 23-\mathrm{C} 24$

$\mathrm{C} 22-\mathrm{C} 23-\mathrm{H} 23$

$\mathrm{C} 24-\mathrm{C} 23-\mathrm{H} 23$

$\mathrm{C} 23-\mathrm{C} 24-\mathrm{C} 19$

$\mathrm{C} 23-\mathrm{C} 24-\mathrm{H} 24$

$\mathrm{C} 19-\mathrm{C} 24-\mathrm{H} 24$

$\mathrm{N} 5-\mathrm{C} 25-\mathrm{C} 26$

$\mathrm{N} 5-\mathrm{C} 25-\mathrm{H} 25$

$\mathrm{C} 26-\mathrm{C} 25-\mathrm{H} 25$

$\mathrm{C} 27-\mathrm{C} 26-\mathrm{C} 31$

$\mathrm{C} 27-\mathrm{C} 26-\mathrm{C} 25$
$1.484(8)$

0.9700

0.9700

$1.475(8)$

0.9700

0.9700

$1.488(7)$

0.9700

0.9700

0.9700

0.9700

$120.9(2)$

$107.5(2)$

$104.2(2)$

107.9 (2)

$108.8(2)$

106.7 (2)

$114.9(3)$

$120(4)$

111 (4)

$115.2(4)$

117.4 (4)

$119.0(5)$

$113.3(5)$

$120.7(5)$

$120.6(4)$

$118.6(4)$

119.5 (5)

120.3

120.3

119.1 (5)

120.5

120.5

$121.7(5)$

119.2 (5)

$119.0(5)$

119.8 (5)

120.1

120.1

119.2 (5)

120.4

120.4

121.4 (5)

119.3

119.3

$116.3(5)$

$123.7(5)$ 


\begin{tabular}{|c|c|c|c|}
\hline $\mathrm{C} 13-\mathrm{C} 8-\mathrm{C} 7$ & $120.2(5)$ & $\mathrm{C} 31-\mathrm{C} 26-\mathrm{C} 25$ & $120.0(5)$ \\
\hline $\mathrm{C} 10-\mathrm{C} 9-\mathrm{C} 8$ & $122.3(5)$ & $\mathrm{C} 28-\mathrm{C} 27-\mathrm{C} 26$ & $122.4(5)$ \\
\hline $\mathrm{C} 10-\mathrm{C} 9-\mathrm{H} 9$ & 118.8 & $\mathrm{C} 28-\mathrm{C} 27-\mathrm{H} 27$ & 118.8 \\
\hline $\mathrm{C} 8-\mathrm{C} 9-\mathrm{H} 9$ & 118.8 & $\mathrm{C} 26-\mathrm{C} 27-\mathrm{H} 27$ & 118.8 \\
\hline $\mathrm{C} 9-\mathrm{C} 10-\mathrm{C} 11$ & $120.6(5)$ & $\mathrm{C} 27-\mathrm{C} 28-\mathrm{C} 29$ & $121.0(5)$ \\
\hline $\mathrm{C} 9-\mathrm{C} 10-\mathrm{H} 10$ & 119.7 & $\mathrm{C} 27-\mathrm{C} 28-\mathrm{H} 28$ & 119.5 \\
\hline $\mathrm{C} 11-\mathrm{C} 10-\mathrm{H} 10$ & 119.7 & $\mathrm{C} 29-\mathrm{C} 28-\mathrm{H} 28$ & 119.5 \\
\hline $\mathrm{C} 12-\mathrm{C} 11-\mathrm{C} 10$ & $116.8(5)$ & $\mathrm{C} 30-\mathrm{C} 29-\mathrm{N} 6$ & $121.7(5)$ \\
\hline $\mathrm{C} 12-\mathrm{C} 11-\mathrm{N} 3$ & $123.6(5)$ & $\mathrm{C} 30-\mathrm{C} 29-\mathrm{C} 28$ & $116.4(5)$ \\
\hline $\mathrm{C} 10-\mathrm{C} 11-\mathrm{N} 3$ & $119.6(5)$ & $\mathrm{N} 6-\mathrm{C} 29-\mathrm{C} 28$ & $121.9(5)$ \\
\hline $\mathrm{C} 13-\mathrm{C} 12-\mathrm{C} 11$ & $121.9(5)$ & $\mathrm{C} 31-\mathrm{C} 30-\mathrm{C} 29$ & $122.2(5)$ \\
\hline $\mathrm{C} 13-\mathrm{C} 12-\mathrm{H} 12$ & 119.1 & $\mathrm{C} 31-\mathrm{C} 30-\mathrm{H} 30$ & 118.9 \\
\hline $\mathrm{C} 11-\mathrm{C} 12-\mathrm{H} 12$ & 119.1 & $\mathrm{C} 29-\mathrm{C} 30-\mathrm{H} 30$ & 118.9 \\
\hline $\mathrm{C} 12-\mathrm{C} 13-\mathrm{C} 8$ & $121.3(5)$ & $\mathrm{C} 30-\mathrm{C} 31-\mathrm{C} 26$ & $121.8(5)$ \\
\hline $\mathrm{C} 12-\mathrm{C} 13-\mathrm{H} 13$ & 119.3 & $\mathrm{C} 30-\mathrm{C} 31-\mathrm{H} 31$ & 119.1 \\
\hline $\mathrm{C} 8-\mathrm{C} 13-\mathrm{H} 13$ & 119.3 & $\mathrm{C} 26-\mathrm{C} 31-\mathrm{H} 31$ & 119.1 \\
\hline $\mathrm{N} 3-\mathrm{C} 14-\mathrm{C} 15$ & $120.8(6)$ & $\mathrm{N} 6-\mathrm{C} 32-\mathrm{C} 33$ & $114.2(5)$ \\
\hline $\mathrm{N} 3-\mathrm{C} 14-\mathrm{H} 14 \mathrm{~A}$ & 107.1 & $\mathrm{~N} 6-\mathrm{C} 32-\mathrm{H} 32 \mathrm{~A}$ & 108.7 \\
\hline $\mathrm{C} 15-\mathrm{C} 14-\mathrm{H} 14 \mathrm{~A}$ & 107.1 & $\mathrm{C} 33-\mathrm{C} 32-\mathrm{H} 32 \mathrm{~A}$ & 108.7 \\
\hline N3-C14-H14B & 107.1 & N6-C32-H32B & 108.7 \\
\hline $\mathrm{C} 15-\mathrm{C} 14-\mathrm{H} 14 \mathrm{~B}$ & 107.1 & $\mathrm{C} 33-\mathrm{C} 32-\mathrm{H} 32 \mathrm{~B}$ & 108.7 \\
\hline $\mathrm{H} 14 \mathrm{~A}-\mathrm{C} 14-\mathrm{H} 14 \mathrm{~B}$ & 106.8 & $\mathrm{H} 32 \mathrm{~A}-\mathrm{C} 32-\mathrm{H} 32 \mathrm{~B}$ & 107.6 \\
\hline $\mathrm{C} 16-\mathrm{C} 15-\mathrm{C} 14$ & $116.7(6)$ & $\mathrm{C} 34-\mathrm{C} 33-\mathrm{C} 32$ & $113.3(6)$ \\
\hline $\mathrm{C} 16-\mathrm{C} 15-\mathrm{H} 15 \mathrm{~A}$ & 108.1 & $\mathrm{C} 34-\mathrm{C} 33-\mathrm{H} 33 \mathrm{~A}$ & 108.9 \\
\hline $\mathrm{C} 14-\mathrm{C} 15-\mathrm{H} 15 \mathrm{~A}$ & 108.1 & $\mathrm{C} 32-\mathrm{C} 33-\mathrm{H} 33 \mathrm{~A}$ & 108.9 \\
\hline $\mathrm{C} 16-\mathrm{C} 15-\mathrm{H} 15 \mathrm{~B}$ & 108.1 & $\mathrm{C} 34-\mathrm{C} 33-\mathrm{H} 33 \mathrm{~B}$ & 108.9 \\
\hline $\mathrm{C} 14-\mathrm{C} 15-\mathrm{H} 15 \mathrm{~B}$ & 108.1 & $\mathrm{C} 32-\mathrm{C} 33-\mathrm{H} 33 \mathrm{~B}$ & 108.9 \\
\hline $\mathrm{H} 15 \mathrm{~A}-\mathrm{C} 15-\mathrm{H} 15 \mathrm{~B}$ & 107.3 & $\mathrm{H} 33 \mathrm{~A}-\mathrm{C} 33-\mathrm{H} 33 \mathrm{~B}$ & 107.7 \\
\hline $\mathrm{C} 17-\mathrm{C} 16-\mathrm{C} 15$ & $114.7(6)$ & $\mathrm{C} 35-\mathrm{C} 34-\mathrm{C} 33$ & $110.9(6)$ \\
\hline $\mathrm{C} 17-\mathrm{C} 16-\mathrm{H} 16 \mathrm{~A}$ & 108.6 & $\mathrm{C} 35-\mathrm{C} 34-\mathrm{H} 34 \mathrm{~A}$ & 109.5 \\
\hline $\mathrm{C} 15-\mathrm{C} 16-\mathrm{H} 16 \mathrm{~A}$ & 108.6 & $\mathrm{C} 33-\mathrm{C} 34-\mathrm{H} 34 \mathrm{~A}$ & 109.5 \\
\hline $\mathrm{C} 17-\mathrm{C} 16-\mathrm{H} 16 \mathrm{~B}$ & 108.6 & $\mathrm{C} 35-\mathrm{C} 34-\mathrm{H} 34 \mathrm{~B}$ & 109.5 \\
\hline $\mathrm{C} 15-\mathrm{C} 16-\mathrm{H} 16 \mathrm{~B}$ & 108.6 & $\mathrm{C} 33-\mathrm{C} 34-\mathrm{H} 34 \mathrm{~B}$ & 109.5 \\
\hline $\mathrm{H} 16 \mathrm{~A}-\mathrm{C} 16-\mathrm{H} 16 \mathrm{~B}$ & 107.6 & $\mathrm{H} 34 \mathrm{~A}-\mathrm{C} 34-\mathrm{H} 34 \mathrm{~B}$ & 108.1 \\
\hline $\mathrm{C} 16-\mathrm{C} 17-\mathrm{C} 18$ & $119.4(6)$ & $\mathrm{C} 34-\mathrm{C} 35-\mathrm{C} 36$ & $112.8(6)$ \\
\hline $\mathrm{C} 16-\mathrm{C} 17-\mathrm{H} 17 \mathrm{~A}$ & 107.5 & $\mathrm{C} 34-\mathrm{C} 35-\mathrm{H} 35 \mathrm{~A}$ & 109.0 \\
\hline $\mathrm{C} 18-\mathrm{C} 17-\mathrm{H} 17 \mathrm{~A}$ & 107.5 & $\mathrm{C} 36-\mathrm{C} 35-\mathrm{H} 35 \mathrm{~A}$ & 109.0 \\
\hline $\mathrm{C} 16-\mathrm{C} 17-\mathrm{H} 17 \mathrm{~B}$ & 107.5 & $\mathrm{C} 34-\mathrm{C} 35-\mathrm{H} 35 \mathrm{~B}$ & 109.0 \\
\hline $\mathrm{C} 18-\mathrm{C} 17-\mathrm{H} 17 \mathrm{~B}$ & 107.5 & $\mathrm{C} 36-\mathrm{C} 35-\mathrm{H} 35 \mathrm{~B}$ & 109.0 \\
\hline $\mathrm{H} 17 \mathrm{~A}-\mathrm{C} 17-\mathrm{H} 17 \mathrm{~B}$ & 107.0 & $\mathrm{H} 35 \mathrm{~A}-\mathrm{C} 35-\mathrm{H} 35 \mathrm{~B}$ & 107.8 \\
\hline $\mathrm{N} 3-\mathrm{C} 18-\mathrm{C} 17$ & $116.9(5)$ & $\mathrm{N} 6-\mathrm{C} 36-\mathrm{C} 35$ & $115.6(5)$ \\
\hline $\mathrm{N} 3-\mathrm{C} 18-\mathrm{H} 18 \mathrm{~A}$ & 108.1 & N6-C36- H36A & 108.4 \\
\hline $\mathrm{C} 17-\mathrm{C} 18-\mathrm{H} 18 \mathrm{~A}$ & 108.1 & $\mathrm{C} 35-\mathrm{C} 36-\mathrm{H} 36 \mathrm{~A}$ & 108.4 \\
\hline $\mathrm{N} 3-\mathrm{C} 18-\mathrm{H} 18 \mathrm{~B}$ & 108.1 & $\mathrm{~N} 6-\mathrm{C} 36-\mathrm{H} 36 \mathrm{~B}$ & 108.4 \\
\hline $\mathrm{C} 17-\mathrm{C} 18-\mathrm{H} 18 \mathrm{~B}$ & 108.1 & $\mathrm{C} 35-\mathrm{C} 36-\mathrm{H} 36 \mathrm{~B}$ & 108.4 \\
\hline $\mathrm{H} 18 \mathrm{~A}-\mathrm{C} 18-\mathrm{H} 18 \mathrm{~B}$ & 107.3 & $\mathrm{H} 36 \mathrm{~A}-\mathrm{C} 36-\mathrm{H} 36 \mathrm{~B}$ & 107.4 \\
\hline $\mathrm{O} 1-\mathrm{S} 1-\mathrm{N} 1-\mathrm{N} 2$ & $175.8(3)$ & $\mathrm{O} 4-\mathrm{S} 2-\mathrm{N} 4-\mathrm{N} 5$ & $-47.7(4)$ \\
\hline
\end{tabular}




\begin{tabular}{|c|c|}
\hline $\mathrm{O} 2-\mathrm{S} 1-\mathrm{N} 1-\mathrm{N} 2$ & $-55.0(4)$ \\
\hline $\mathrm{C} 1-\mathrm{S} 1-\mathrm{N} 1-\mathrm{N} 2$ & $59.7(4)$ \\
\hline $\mathrm{S} 1-\mathrm{N} 1-\mathrm{N} 2-\mathrm{C} 7$ & $-163.0(3)$ \\
\hline $\mathrm{O} 1-\mathrm{S} 1-\mathrm{C} 1-\mathrm{C} 2$ & $159.7(4)$ \\
\hline $\mathrm{O} 2-\mathrm{S} 1-\mathrm{C} 1-\mathrm{C} 2$ & $26.7(5)$ \\
\hline $\mathrm{N} 1-\mathrm{S} 1-\mathrm{C} 1-\mathrm{C} 2$ & $-87.9(4)$ \\
\hline $\mathrm{O} 1-\mathrm{S} 1-\mathrm{C} 1-\mathrm{C} 6$ & $-22.4(5)$ \\
\hline $\mathrm{O} 2-\mathrm{S} 1-\mathrm{C} 1-\mathrm{C} 6$ & $-155.4(4)$ \\
\hline $\mathrm{N} 1-\mathrm{S} 1-\mathrm{C} 1-\mathrm{C} 6$ & $90.0(4)$ \\
\hline $\mathrm{C} 6-\mathrm{C} 1-\mathrm{C} 2-\mathrm{C} 3$ & $-0.3(8)$ \\
\hline $\mathrm{S} 1-\mathrm{C} 1-\mathrm{C} 2-\mathrm{C} 3$ & $177.6(4)$ \\
\hline $\mathrm{C} 1-\mathrm{C} 2-\mathrm{C} 3-\mathrm{C} 4$ & $-1.4(9)$ \\
\hline $\mathrm{C} 2-\mathrm{C} 3-\mathrm{C} 4-\mathrm{C} 5$ & $3.3(9)$ \\
\hline $\mathrm{C} 2-\mathrm{C} 3-\mathrm{C} 4-\mathrm{Cl} 1$ & $-178.1(5)$ \\
\hline $\mathrm{C} 3-\mathrm{C} 4-\mathrm{C} 5-\mathrm{C} 6$ & $-3.5(9)$ \\
\hline $\mathrm{C} 11-\mathrm{C} 4-\mathrm{C} 5-\mathrm{C} 6$ & $177.9(4)$ \\
\hline $\mathrm{C} 2-\mathrm{C} 1-\mathrm{C} 6-\mathrm{C} 5$ & $0.1(8)$ \\
\hline $\mathrm{S} 1-\mathrm{C} 1-\mathrm{C} 6-\mathrm{C} 5$ & $-177.9(4)$ \\
\hline $\mathrm{C} 4-\mathrm{C} 5-\mathrm{C} 6-\mathrm{C} 1$ & $1.8(9)$ \\
\hline $\mathrm{N} 1-\mathrm{N} 2-\mathrm{C} 7-\mathrm{C} 8$ & $-178.4(4)$ \\
\hline $\mathrm{N} 2-\mathrm{C} 7-\mathrm{C} 8-\mathrm{C} 9$ & $-6.3(7)$ \\
\hline $\mathrm{N} 2-\mathrm{C} 7-\mathrm{C} 8-\mathrm{C} 13$ & $172.8(4)$ \\
\hline $\mathrm{C} 13-\mathrm{C} 8-\mathrm{C} 9-\mathrm{C} 10$ & $-0.7(7)$ \\
\hline $\mathrm{C} 7-\mathrm{C} 8-\mathrm{C} 9-\mathrm{C} 10$ & $178.5(5)$ \\
\hline $\mathrm{C} 8-\mathrm{C} 9-\mathrm{C} 10-\mathrm{C} 11$ & $0.4(8)$ \\
\hline $\mathrm{C} 9-\mathrm{C} 10-\mathrm{C} 11-\mathrm{C} 12$ & $-0.3(7)$ \\
\hline $\mathrm{C} 9-\mathrm{C} 10-\mathrm{C} 11-\mathrm{N} 3$ & $-178.7(5)$ \\
\hline $\mathrm{C} 14-\mathrm{N} 3-\mathrm{C} 11-\mathrm{C} 12$ & $142.9(6)$ \\
\hline $\mathrm{C} 18-\mathrm{N} 3-\mathrm{C} 11-\mathrm{C} 12$ & $-11.8(7)$ \\
\hline $\mathrm{C} 14-\mathrm{N} 3-\mathrm{C} 11-\mathrm{C} 10$ & $-38.8(8)$ \\
\hline $\mathrm{C} 18-\mathrm{N} 3-\mathrm{C} 11-\mathrm{C} 10$ & $166.4(5)$ \\
\hline $\mathrm{C} 10-\mathrm{C} 11-\mathrm{C} 12-\mathrm{C} 13$ & $0.6(7)$ \\
\hline $\mathrm{N} 3-\mathrm{C} 11-\mathrm{C} 12-\mathrm{C} 13$ & $178.9(5)$ \\
\hline $\mathrm{C} 11-\mathrm{C} 12-\mathrm{C} 13-\mathrm{C} 8$ & $-1.0(8)$ \\
\hline $\mathrm{C} 9-\mathrm{C} 8-\mathrm{C} 13-\mathrm{C} 12$ & $1.0(7)$ \\
\hline $\mathrm{C} 7-\mathrm{C} 8-\mathrm{C} 13-\mathrm{C} 12$ & $-178.2(5)$ \\
\hline $\mathrm{C} 11-\mathrm{N} 3-\mathrm{C} 14-\mathrm{C} 15$ & $174.4(7)$ \\
\hline $\mathrm{C} 18-\mathrm{N} 3-\mathrm{C} 14-\mathrm{C} 15$ & $-30.4(10)$ \\
\hline $\mathrm{N} 3-\mathrm{C} 14-\mathrm{C} 15-\mathrm{C} 16$ & $30.9(12)$ \\
\hline $\mathrm{C} 14-\mathrm{C} 15-\mathrm{C} 16-\mathrm{C} 17$ & $-30.7(11)$ \\
\hline $\mathrm{C} 15-\mathrm{C} 16-\mathrm{C} 17-\mathrm{C} 18$ & $32.7(12)$ \\
\hline $\mathrm{C} 14-\mathrm{N} 3-\mathrm{C} 18-\mathrm{C} 17$ & $30.2(9)$ \\
\hline $\mathrm{C} 11-\mathrm{N} 3-\mathrm{C} 18-\mathrm{C} 17$ & $-173.8(6)$ \\
\hline $\mathrm{C} 16-\mathrm{C} 17-\mathrm{C} 18-\mathrm{N} 3$ & $-32.9(11)$ \\
\hline
\end{tabular}

\begin{tabular}{|c|c|}
\hline $\mathrm{O} 3-\mathrm{S} 2-\mathrm{N} 4-\mathrm{N} 5$ & $-177.1(3)$ \\
\hline $\mathrm{C} 19-\mathrm{S} 2-\mathrm{N} 4-\mathrm{N} 5$ & $67.8(4)$ \\
\hline $\mathrm{S} 2-\mathrm{N} 4-\mathrm{N} 5-\mathrm{C} 25$ & $-162.9(3)$ \\
\hline $\mathrm{O} 4-\mathrm{S} 2-\mathrm{C} 19-\mathrm{C} 20$ & $26.0(5)$ \\
\hline $\mathrm{O} 3-\mathrm{S} 2-\mathrm{C} 19-\mathrm{C} 20$ & $158.8(4)$ \\
\hline $\mathrm{N} 4-\mathrm{S} 2-\mathrm{C} 19-\mathrm{C} 20$ & $-89.3(4)$ \\
\hline $\mathrm{O} 4-\mathrm{S} 2-\mathrm{C} 19-\mathrm{C} 24$ & $-156.9(4)$ \\
\hline $\mathrm{O} 3-\mathrm{S} 2-\mathrm{C} 19-\mathrm{C} 24$ & $-24.1(4)$ \\
\hline $\mathrm{N} 4-\mathrm{S} 2-\mathrm{C} 19-\mathrm{C} 24$ & $87.8(4)$ \\
\hline $\mathrm{C} 24-\mathrm{C} 19-\mathrm{C} 20-\mathrm{C} 21$ & $1.7(7)$ \\
\hline $\mathrm{S} 2-\mathrm{C} 19-\mathrm{C} 20-\mathrm{C} 21$ & $178.7(4)$ \\
\hline $\mathrm{C} 19-\mathrm{C} 20-\mathrm{C} 21-\mathrm{C} 22$ & $-1.9(8)$ \\
\hline $\mathrm{C} 20-\mathrm{C} 21-\mathrm{C} 22-\mathrm{C} 23$ & $1.5(8)$ \\
\hline $\mathrm{C} 20-\mathrm{C} 21-\mathrm{C} 22-\mathrm{C} 2$ & $-179.5(4)$ \\
\hline $\mathrm{C} 21-\mathrm{C} 22-\mathrm{C} 23-\mathrm{C} 24$ & $-1.0(8)$ \\
\hline $\mathrm{C} 2-\mathrm{C} 22-\mathrm{C} 23-\mathrm{C} 24$ & $-180.0(4)$ \\
\hline $\mathrm{C} 22-\mathrm{C} 23-\mathrm{C} 24-\mathrm{C} 19$ & $0.8(8)$ \\
\hline $\mathrm{C} 20-\mathrm{C} 19-\mathrm{C} 24-\mathrm{C} 23$ & $-1.1(7)$ \\
\hline $\mathrm{S} 2-\mathrm{C} 19-\mathrm{C} 24-\mathrm{C} 23$ & $-178.2(4)$ \\
\hline $\mathrm{N} 4-\mathrm{N} 5-\mathrm{C} 25-\mathrm{C} 26$ & $-175.5(4)$ \\
\hline N5-C25-C26-C27 & $-15.6(7)$ \\
\hline $\mathrm{N} 5-\mathrm{C} 25-\mathrm{C} 26-\mathrm{C} 31$ & $161.8(4)$ \\
\hline $\mathrm{C} 31-\mathrm{C} 26-\mathrm{C} 27-\mathrm{C} 28$ & $-0.1(7)$ \\
\hline $\mathrm{C} 25-\mathrm{C} 26-\mathrm{C} 27-\mathrm{C} 28$ & $177.4(4)$ \\
\hline $\mathrm{C} 26-\mathrm{C} 27-\mathrm{C} 28-\mathrm{C} 29$ & $-0.7(7)$ \\
\hline $\mathrm{C} 36-\mathrm{N} 6-\mathrm{C} 29-\mathrm{C} 30$ & $-35.3(7)$ \\
\hline $\mathrm{C} 32-\mathrm{N} 6-\mathrm{C} 29-\mathrm{C} 30$ & $-178.2(5)$ \\
\hline $\mathrm{C} 36-\mathrm{N} 6-\mathrm{C} 29-\mathrm{C} 28$ & $148.0(5)$ \\
\hline $\mathrm{C} 32-\mathrm{N} 6-\mathrm{C} 29-\mathrm{C} 28$ & $5.1(7)$ \\
\hline $\mathrm{C} 27-\mathrm{C} 28-\mathrm{C} 29-\mathrm{C} 30$ & $0.3(7)$ \\
\hline $\mathrm{C} 27-\mathrm{C} 28-\mathrm{C} 29-\mathrm{N} 6$ & $177.3(4)$ \\
\hline $\mathrm{N} 6-\mathrm{C} 29-\mathrm{C} 30-\mathrm{C} 31$ & $-176.1(4)$ \\
\hline $\mathrm{C} 28-\mathrm{C} 29-\mathrm{C} 30-\mathrm{C} 31$ & $0.9(7)$ \\
\hline $\mathrm{C} 29-\mathrm{C} 30-\mathrm{C} 31-\mathrm{C} 26$ & $-1.7(8)$ \\
\hline $\mathrm{C} 27-\mathrm{C} 26-\mathrm{C} 31-\mathrm{C} 30$ & $1.3(7)$ \\
\hline $\mathrm{C} 25-\mathrm{C} 26-\mathrm{C} 31-\mathrm{C} 30$ & $-176.3(4)$ \\
\hline $\mathrm{C} 29-\mathrm{N} 6-\mathrm{C} 32-\mathrm{C} 33$ & $-168.9(5)$ \\
\hline $\mathrm{C} 36-\mathrm{N} 6-\mathrm{C} 32-\mathrm{C} 33$ & $46.7(7)$ \\
\hline $\mathrm{N} 6-\mathrm{C} 32-\mathrm{C} 33-\mathrm{C} 34$ & $-49.6(8)$ \\
\hline $\mathrm{C} 32-\mathrm{C} 33-\mathrm{C} 34-\mathrm{C} 35$ & $50.7(8)$ \\
\hline $\mathrm{C} 33-\mathrm{C} 34-\mathrm{C} 35-\mathrm{C} 36$ & $-50.0(8)$ \\
\hline $\mathrm{C} 29-\mathrm{N} 6-\mathrm{C} 36-\mathrm{C} 35$ & $168.0(5)$ \\
\hline $\mathrm{C} 32-\mathrm{N} 6-\mathrm{C} 36-\mathrm{C} 35$ & $-47.1(8)$ \\
\hline $\mathrm{C} 34-\mathrm{C} 35-\mathrm{C} 36-\mathrm{N} 6$ & $49.5(8)$ \\
\hline
\end{tabular}


Hydrogen-bond geometry $\left(\AA,{ }^{\circ}\right)$

\begin{tabular}{lllll}
\hline$D-\mathrm{H} \cdots A$ & $D-\mathrm{H}$ & $\mathrm{H} \cdots A$ & $D \cdots A$ & $D-\mathrm{H} \cdots A$ \\
\hline $\mathrm{N} 1-\mathrm{H} 1 N \cdots \mathrm{O} 2^{\mathrm{i}}$ & $0.83(4)$ & $2.26(5)$ & $3.025(5)$ & $153(5)$ \\
$\mathrm{N} 4-\mathrm{H} 4 N \cdots \mathrm{O} 4^{\mathrm{ii}}$ & $0.84(5)$ & $2.29(5)$ & $3.115(6)$ & $169(5)$ \\
\hline
\end{tabular}

Symmetry codes: (i) $x, y+1, z$; (ii) $x, y-1, z$. 